Ecole d'Eté Systèmes Optiques

\title{
Radars laser
}

\author{
J.L. Meyzonnette
}

Institut d'Optique Théorique et Appliquée, E.S.O., Centre Universitaire d'Orsay, Bat. 503, BP. 147, F-91403 Orsay cedex, France

\section{PLAN}

INTRODUCTION : Radars et Lidars

CHAPITRE I : ELEMENTS DE CONCEPTION

I.1 CONSIDERATIONS GENERALES

1.2 PARAMETRES DE CONCEPTION

1.3 PERFORMANCES D'UN RADAR LASER

I.3.1 Calcul du signal laser

1.3.2 Bilan de portée (général)

1.3.3 Cas d'un radar laser cohérent

CHAPITRE II : TECHNIQUES DE MESURES

II.1 TELEMETRIE LASER (Détection directe)

II.2 MESURE DE VITESSE RADIALE

II.3 TELEMETRIE DOPPLER (Détection hétérodyne)

II.3.1 Impulsions fines

II.3.2 Emission continue et modulation de fréquence

I1.4 ECARTOMETRIE LASER

II.5 PRECISION DES MESURES

CHAPITRE III : EXEMPLE D'APPLICATIONS DE RADARS OPTIQUES

III.1 LIDAR ATMOSPHERIQUE

III.1.1 Généralites

III.1.2 Lidar à absorption différentielle (DIAL)

III.1.3 Lidar atmosphérique Doppler

III.2 RADAR LASER POUR DETECTION DE CABLES

III.3 RADAR LASER MULTIFONCTION

\section{INTRODUCTION}

On assiste, depuis quelques années, à un rapprochement entre les domaines de l'optique et du radar, qui se manifeste non seulement au point de vue spectral (millimétrique d'un côté, infrarouge de l'autre) mais aussi et surtout dans la conception et le mode de fonctionnement des systèmes.

C'est ainsi que, grâce aux progrès réalisés en particulier sur les sources et les récepteurs optiques, on voit apparaître un nombre grandissant de systèmes optiques, dont l'architecture, les techniques d'émission, de réception et de traitement du signal sont directement dérivées du radar. Ces systèmes, dénommés radars optiques, ou radars laser, ou lidars, etc... se développent grâce à linvention et aux progrès des lasers, dont ils exploitent les propriétés spatiales, spectrales et temporelles.

Le présent texte se propose de décrire œ type de système optique : le chapitre I présente les éléments essentiels qui interviennent dans la conception, le chapitre II concerne les principales possibilités de mesure avec les techniques correspondantes. Enfin, le dernier chapitre, consacré aux applications. illustre les possibilités des lidars en sondage atmosphérique, détection de câbles et conduite de tir. 
CHAPITRE I

\section{ELEMENTS DE CONCEPTION}

\section{I.1. CONSIDERATIONS GENERALES}

Par définition, un lidar (ou radar optique) est un radar dont l'émission se situe dans le domaine optique : il émet un faisceau optique (généralement à partir d'un laser), en direction de l'objet à localiser, et collecte une fraction du flux diffusé par œ dernier, à partir de laquelle sont reconstituées les informations recherchées (distance, vitesse, réflectance, densité).

Emetteur et récepteur optiques peuvent être soit liés l'un à l'autre (systèmes optiques identiques ou accolés : lidars dits monostatiques), soit indépendants l'un de l'autre (lidars bistatiques), La Figure I.1 illustre ces 2 configurations, dans lesquelles les champs des systèmes optiques à l'émission et à la réception présentent une zone commune plus ou moins importante, au sein de laquelle doit se trouver l'objet à détecter.

Dans l'une ou l'autre configuration, le radar optique illumine donc la scène, ou la cible, grâce à un pinceau lumineux étroit et capte en retour le flux réfléchi ou diffusé par l'objet grâce au récepteur, dont l'axe est pointé sur l'objet.

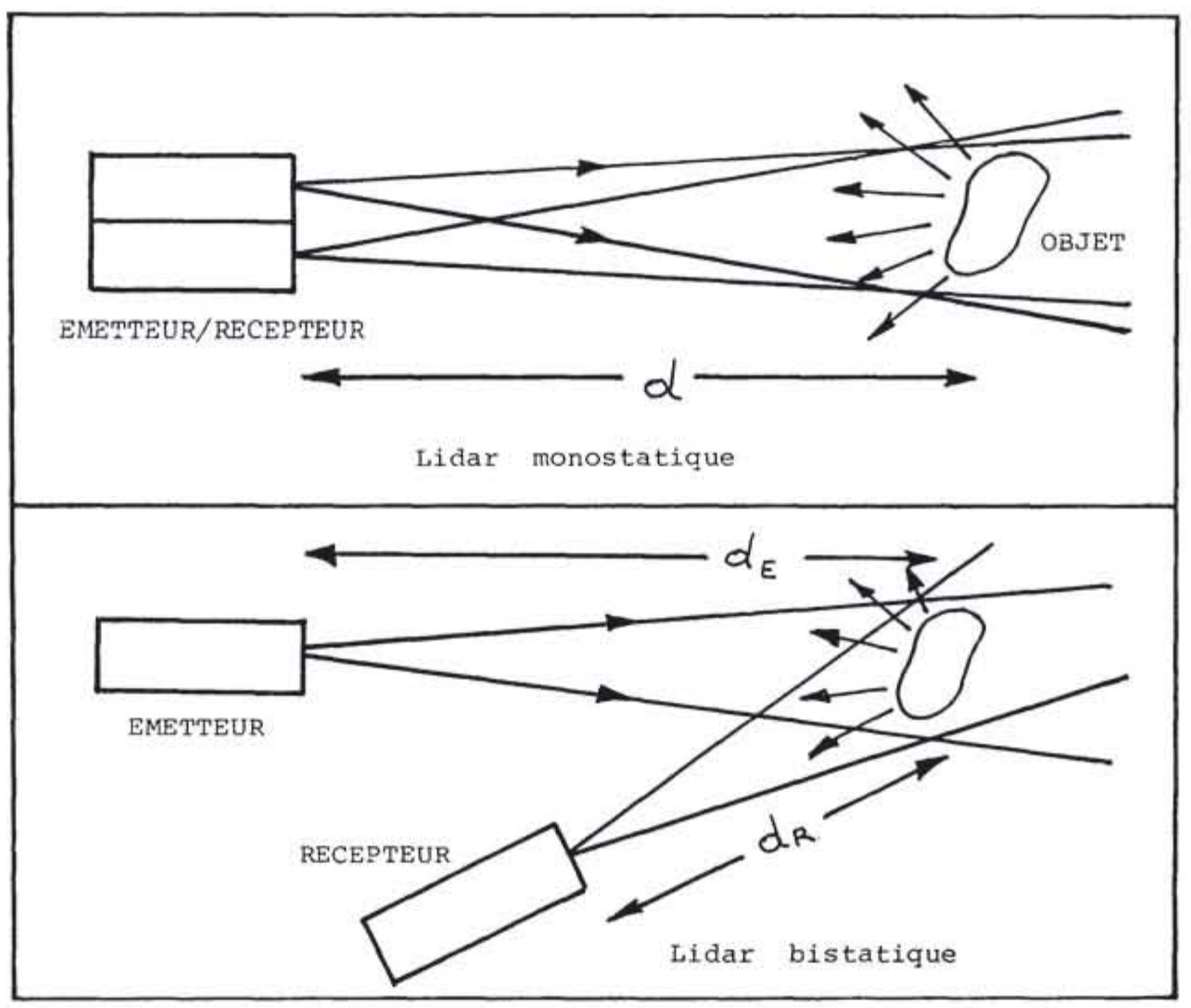

Figure 1-1: Configurations générales d'un radar optique 
Le schéma de la figure 1.2 représente l'architecture typique d'un radar optique, avec ses principaux composants.

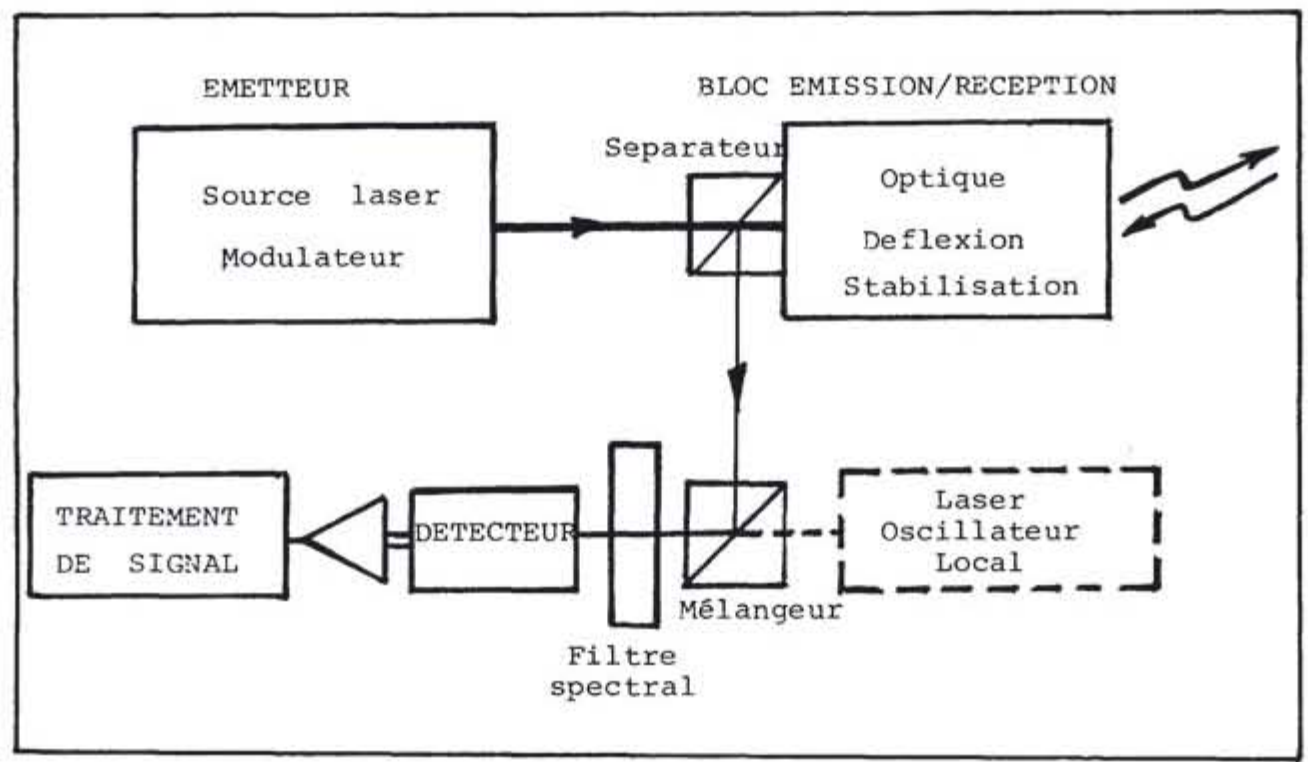

Fig 1-2 : Architecture et composants de base d'un radar optique

En suivant le cheminement des photons et de linformation dans le système, on notera les sous-ensembles ci-dessous :

- La source (laser) avec son électronique de commande, et, éventuellement, un modulateur de faisceau (en amplitude, phase ou fréquence) intégré ou non à la source elle-même.

- Un séparateur de faisceau dans le cas de systèmes monostatiques à optique d'émission-réception commune.

- Les optiques d'émission et de réception (communes ou non)

- Dans la plupart des cas, un dispositif d'adressage angulaire de la ligne de visée, qui assure soit une déflexion aléatoire (en fonction de commandes extérieures) soit un balayage systématique du faisceau émis. Suivant les applications, œe dispositif peut être complété par un système de stabilisation.

- Un ensemble de filtres, adaptés spatialement et spectralement au signal lumineux en provenance de l'objet et destinés à réduire les sources de parasitage optique du détecteur.

- Un photodétecteur, dont le rôle est de convertir en signal électrique la fraction du flux laser diffusé par l'objet, qui est captée par l'optique de collection (conversion obtenue par absorption de ce flux). Le détecteur est généralement muni d'une électronique de mise en oeuvre (tensions de polarisation), d'une électronique de préamplification du signal, de dispositifs de mise en froid (en particulier dans le cas des lidars infrarouges).

- Une électronique de traitement du signal (analogique et numérique) et de commande des servomécanismes.

- Des dispositifs d'exploitation automatique et/ou de visualisation des résultats. 


\subsection{PARAMETRES DE CONCEPTION}

Le concepteur d'un radar laser cherchera à optimiser les performances de son système en fonction de nombreux paramètres qu'il aura soin d'évaluer au mieux, et parmi lesquels on peut citer :

- Les propriétés angulaires et spectrales, en diffusion ou réflexion, de l'objet visé

- Les propriétés spectrales en transmission (absorption, diffusion, turbulence) du milieu de propagation.

- Les caractéristiques de la source : choix de la longueur d'onde, émission continue ou impulsionnelle, stabilité en fréquence, etc...

- Choix du mode de détection (directe ou hétérodyne) et du type de traitement du signal, en fonction de l'information recherchée.

Pour une mission donnée, l'optimisation des performances du système passe toujours par l'amélioration du rapport signal à bruit en sortie du détecteur. II est donc essentiel d'optimiser le flux laser détecté par le système et, pour cela, de bien choisir l'illuminateur, en fonction des contraintes dues à l'objet et au milieu de transmission.

L'utilisation d'un laser comme émetteur s'impose dans la plupart des cas, parce que ce demier présente vis-â-vis des sources conventionnelles des avantages considérables en finesse angulaire (éclairage sélectif de l'objet vis-à-vis du fond), spectrales (possibilités de filtrage) et temporelle (obtention d'impulsions fines, grandes possibilités de modulation du faisceau).

Le milieu de propagation joue lui-aussi un rôle primordial, en particulier si les distances à parcourir sont importantes. Dans le cas particulier de l'atmosphère, on rappelle que cette dernière présente des domaines de transmission ("fenêtres atmosphériques") définis par les bandes d'absorption des différents gaz qui la composent $\left(\mathrm{H}_{2} \mathrm{O}, \mathrm{O}_{2}, \mathrm{CO}_{2}, \mathrm{~N}_{2}, \ldots\right)$. Ces fenêtres imposent des restrictions sur le choix de la longueur d'onde d'émission du laser, compte tenu des conditions réelles d'utilisation du système (humidité, température, pression, type et concent:ation des aérosols, poussières, fumées, etc....).

A cause de la finesse spectrale des raies laser, on prendra soin de ne pas utiliser aveuglément les tableaux de transmission atmosphérique généralement consultés pour la conception des systèmes optiques passifs et qui ne sont valides que pour des bandes spectrales larges (par exemple, code Lowtran). On s'appuiera donc sur des logiciels spécialisés pour raies laser, tels que le Fascode (développé par AFGL, Air Force Geophysics Laboratory).

En plus de ces effets d'atténuation du faisceau, le milieu peut perturber la propagation du faisceau par ses inhomogénéités en indice de réfraction (turbulence), de façon d'autant plus grave que les fluctuations de température sont grandes et la longueur d'onde du laser utilisé petite.

\section{I.3. PERFOMANCES D'UN RADAR LASER}

De façon générale, l'évalużtion des performances d'un radar laser conceme l'ensemble ou une partie des points suivants :

- Portée, c'est-à-dire la distance limite au-delà de laquelle le système ne remplit plus de façon satisfaisante les fonctions demandées.

- Précision des mesures, par exemple, en distances, vitesses ou angles.

- Résolution, c'est-à-dire l'aptitude à discerner la présence de plusieurs cibles proches l'une de l'autre en distance, vitesse ou direction

- Cadence d'information

- Poids, Volume, Prix, etc... 
On peut appliquer à un radar optique les procédures généralement suivies pour la conception et l'évaluation des radars dits conventionnels avec cependant quelques spécificités dues aux différences de longueurs d'onde et qui portent essentiellement sur :

- le comportement des objets en réflexion

- la transmission de l'atmosphère

- la finesse angulaire du faisceau

On donne ci-dessous une procédure couramment employée pour évaluer le bilan de portée d'un radar optique sur objet "solide", basée sur le calcul du signal laser en provenance de l'objet considéré et sur la détermination du rapport signal à bruit en fonction de la distance.

\subsubsection{Calcul du signal laser}

Le calcul du signal laser s'effectue à partir des lois générales de la photométrie géométrique. Par exemple, si l'objet est un diffuseur plan lambertien étendu (c'est-à-dire de dimensions supérieures à celles de la tache laser), de facteur spectral de réflexion diffuse $\rho \lambda$, le flux laser capté en retour par le détecteur est donné par l'expression :

$$
F_{R}=\rho_{\lambda} F_{L} T_{E} T_{R} S_{o p} T_{a t m}(2 d) / \pi d^{2}
$$

oủ $\mathrm{FL} \quad$ : Flux de l'émetteur laser

$T_{\text {atm, }} T_{E}, T_{R} \quad$ : Facteurs de transmission respectifs de l'atmosphère et des optiques d'émission et de réception.

Sop : : Aire de la pupille de réception

d : Distance de l'objet visé

Cette formulation simplifiée suppose que les champs angulaires en émission et réception sont adaptés l'un à l'ac tre (en direction et champ) et en particulier, que le champ en réception ne diaphragme en rien le faisceau laser rétroréfléchi par l'objet.

Dans le cas où le faisceau laser est de dimensions supérieures à celles de l'objet, l'expression ci-dessus peut prendre une forme similaire à "l'équation du radar", par exemple la suivante :

$$
F_{R}=\sigma F_{L} T_{E} T_{R} S_{\infty p} T_{a t m}(2 d) / \theta E^{2} d^{4}
$$

où $\quad \sigma \quad: \quad$ Surface équivalente laser de l'objet (SEL)

$$
\theta E \quad \text { : Angle de divergence totale du faisceau laser à l'émission }
$$

(à l'intérieur de laquelle on suppose l'intensité constante)

Les formules ci-dessus peuvent être résumées de la façon suivante :

$$
F_{R}=k G \rho_{\lambda} F_{L} T_{E} T_{R} S_{\infty \rho} e^{-2 \gamma d} / \pi d^{2}
$$

kG

$=1$ si la dimension de l'objet est supérieure à celle de la tache laser

$=\sigma / \theta E^{2} d^{2} \quad$ si la dimension de l'objet est inférieure à celle de la tache laser

On montre que la surface équivalente laser d'un objet lambertien plan de surface $S$ est liée à sa réflectance diffuse par:

$$
\sigma=S E L=4 \rho S
$$




\subsubsection{Bilan de portée (général)}

A partir du calcul précédent sur la valeur du signal, le bilan de liaison d'un radar laser (ou bilan de portée) sur un objet donné, s'effectue en évaluant la variation du rapport signal à bruit du système à la sortie du détecteur en fonction de la distance de l'objet. La portée est alors la distance de l'objet pour laquelle le rapport signal à bruit atteint une valeur limite imposée par les spécifications du système (en particulier, sur les probabilités de détection et de fausse alarme).

On rappelle que la valeur théorique optimale du rapport signal à bruit s'exprime de 2 façons différentes, suivant que le signal laser est détecté en mode direct (incohérent) ou hétérodyne (cohérent).

$$
\begin{array}{ll}
\text { En détection directe: } & (S / B) d i r=D^{*}(\lambda) F_{R} / \sqrt{A_{d} B} \\
\text { En détection hétérodyne : } & (S / B) \text { het }=\eta(\lambda) F_{R} / h \vee B
\end{array}
$$

où $F_{R}$ est le flux laser de signal (ou flux reçu) et où $D^{\circ}(\lambda), \eta(\lambda)$ et Ad sont respectivement la détectivité spécifique et le rendement quantique du détecteur utilisé (mesurés à la longueur d'onde du laser). Ad son aire sensible, et $\mathrm{B}$ la bande passante du traitement électronique.

Dans la pratique, les performances réelles d'un radar laser sont dictées par les valeurs expérimentales des paramètres de base (puissance d'émission laser, réflectance de l'objet, transmission atmosphérique, qualité optique du capteur, bruits du détecteur et du traitement de signal,...)

Si l'on considère par exemple la réflectance de l'objet, la valeur du coefficient de réflexion à prendre en compte (bidirectionnel pour systèmes bistatiques, de rétroréflexion pour systèmes monostatiques) dépend non seulement de la nature et de la forme de l'objet, mais aussi de l'état de sa surface (lisse, rugueux, sec, humide, présence de dépôts ou de peintures superficiels, etc...), de l'angle sous lequel il se présente. etc... Pour un objet donné, on établira expérimentalement les lois de variation angulaire qui définissent son indicatrice d'intensité en réflexion, ou sa surface équivalente laser. Cette caractérisation est indispensable car elle permet de déduire non seulement le comportement moyen de lobjet sous illumination laser, mais aussi les fluctuations de sa réponse laser dont la connaissance est essentielle pour l'établissement d'un bilan réaliste (probabilités de détection différentes sur cibles "stationnaires" et "fluctuantes").

Comme on l'a signalé plus haut (paragraphe 1.2), le milieu de propagation peut dégrader le faisceau laser, en l'atténuant et en le déformant (turbulence).

Les 2 sources d'atténuation du faisceau pour l'atmosphère (absorption et diffusion du flux par les molécules de gaz et les particules ou aérosols présents dans l'air) ont une importance relative, l'une vis-à-vis de l'autre, qui dépend du domaine spectral du lidar : par exemple, la diffusion par les aérosols est le phénomène prépondérant dans le visible et le proche infrarouge, où la transmission atmosphérique au sol est très fortement corrélée à la distance de visibilité météorologique. Par contre, dans l'infrarouge plus lointain $(\lambda \sim 10 \mu \mathrm{m})$, la diffusion du faisceau laser par les aérosols est faible par rapport à l'absorption due à l'humidité.

En présence de turbulence, les rayons lumineux n'obéissent plus aux lois classiques de la propagation dans les milieux homogènes, ce qui se traduit, pour le faisceau laser, par des :

- Fluctuations angulaires autour de la direction générale de propagation

- Variations sur la divergence (taille du faisceau) et l'éclairement local dans la tache laser

- Fluctuations de phase dans un plan perpendiculaire à la direction de propagation.

Les 2 premières perturbations ci-dessus peuvent affecter tous les types de systèmes laser (cohérents ou non). Les fluctuations de phase affectent plus particulièrement les radars laser cohérents dans la mesure où elles réduisent la cohérence spatiale de l'onde à détecter. 


\subsubsection{Cas d'un radar laser cohérent}

Parce que le récepteur d'un radar laser cohérent est sensible à la phase de l'onde à détecter, les causes de dégradation en performance sont plus nombreuses que dans le cas d'un radar laser à détection directe : un radar laser cohérent ne se comporte pas en simple capteur de flux, mais en interféromètre où toute déformation de l'onde réduit le signal utile.

II en résulte que le calcul du rapport signal à bruit ne doit prendre en compte que cette fraction de signal utilie au récepteur laser hétérodyne, généralement mise sous la forme suivante :

$$
\eta_{h e t}=F U / F_{R}
$$

où $\eta$ het est appelé "rendement d'hétérodynage" et Fu représente le flux utilement détecté.

Sans entrer dans le détail, on peut dire que la valeur de ce rendement d'hétérodynage est dictée par les déformations en phase de l'onde laser qui revient de l'objet, par rapport à une onde théorique, adaptée parfaitement à l'onde oscillateur local et qui sont dues à :

- la cohérence spatiale de l'objet ainsi éclairé (phénomène dit de speckle ou granularité laser)

- la turbulence atmosphérique

- les défauts optiques du montage optique pour le mélange des 2 ondes (aberrations, désalignements, etc...)

Le rendement d'hétérodynage s'exprime généralement sous la forme du produit de plusieurs termes, indépendants les uns des autres et représentatifs des perturbations dues respectivement à l'optique ( $\eta_{\text {optique }}$ ), à l'objet lui-même ( $\eta_{\text {speckle) }}$ ) ou à l'atmosphère ( $\eta_{\text {turbulence }}$ ), soit :

$$
\eta_{\text {het }}=\eta_{\text {optique }} \eta_{\text {speckle }} \eta_{\text {turbulence }}
$$

Ci-dessous, on rappelle quelques unes des dégradations pouvant affecter un système de détection laser hétérodyne :

\section{Théorème de l'antenne}

Parmi les causes possibles de déphasage relatif entre différents points des ondes de signal et d'oscillateur local, on peut citer le décalage angulaire entre 2 ondes théoriquement parfaites (planes). Ce déphasage varie d'autant plus vite que le décalage angulaire est important. On démontre ainsi que le rendement théorique d'un capteur hétérodyne est donné en fonction de l'angle d'incidence de l'onde à détecter, par la courbe de diffraction de la pupille du récepteur ("Théorème de l'Antenne). Ainsi, le champ utile en réception d'un radar laser cohérent se limite-t-il au lobe de diffraction de sa pupille, c'est-à-dire à l'intérieur d'un angle solide approximativement égal à :

où Ap est l'aire de la pupille de réception.

$$
\Omega R \approx \lambda^{2} / A_{p}
$$

Cette caractéristique différencie fortement lidars cohérents et incohérents, car pour ces derniers, les valeurs de champ et d'ouverture dont découplées l'une de lautre. La figure 1.3 montre la courbe de rendement d'hétérodynage théorique d'un récepteur laser hétérodyne en fonction de l'angle d'incidence du faisceau. 


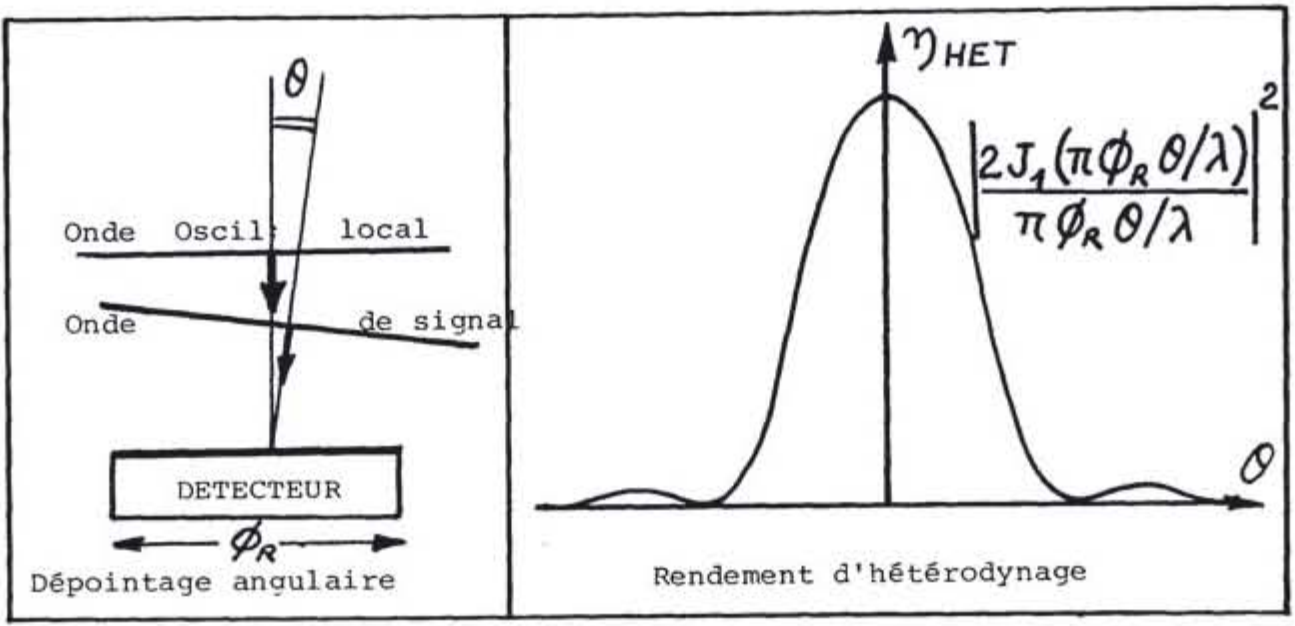

Figure 1.3 : Théorème de l'Antenne

En conséquence, pour adapter au mieux le diagramme d'émission à celui de la réception et éviter au système d'éclairer inutilement des zones de l'espace non "vues" par le récepteur, la plupart des systèmes laser cohérents possèdent des pupilles d'émission et de réception de diamètres similaires (ou identiques).

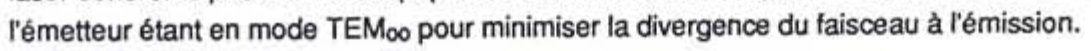

\section{Influence des aberrations}

Une autre cause de désadaptation en phase entre l'onde de signal tt l'oscillateur local est la présence d'aberrations optiques dans le système d'émission/réception : d'une part, les défauts optiques de l'émetteur accroissent la divergence du faisceau laser illuminateur, pour un diamètre de pupille donné, par rapport à la limite théorique imposée par la diffraction. Cela entraine une réduction de l'éclairage sur l'axe au profit de zones hors d'axe moins efficaces en réception, d'après le théorème de l'antenne.

De plus, les aberrations de l'optique de réception déforment la surface d'onde du faisceau laser de retour : si les variances des écarts à la surface d'onde sont respectivement $\Delta \mathrm{em}^{2}$ et $\Delta \Delta_{\text {rec }}{ }^{2}$, à l'émission et à la réception, le rendement d'hétérodynage d'un système laser cohérent pour un objet ponctuel sur l'axe devient, en présence d'aberrations :

$$
\eta \text { Aberrations }=1-4 \pi^{2}\left(\Delta_{\text {em }}^{2}+\Delta_{\text {rec }}^{2}\right) / \lambda^{2}
$$

Si l'on désire maintenir les pertes en rapport signal à bruit, dues aux aberrations du système optique, inférieures à un seuil de l'ordre de $20 \%$ par rapport à la théorie, le système optique doit être proche de la limite de la diffraction $\left(\Delta_{\text {eff }}<\lambda / 20\right)$

\section{Théorème de Zernicke Van Cittert}

Dans le cas où l'objet éclairé par le radar laser est quasi ponctuel, le rendement en fonction de la position angulaire de l'objet dans le lobe d'émission-réception (donné par le théorème de l'antenne) est maximal sur l'axe avec une valeur de $100 \%$.

Par contre, si l'objet éclairé par le faisceau laser est un diffuseur de grande surface, il renvoit sur le 
système laser un ensemble d'ondelettes dont les directions de propagation (dans la pupille du récepteur) ne sont pas exactement colinéaires et dont les phases sont aléatoires. Le théorème de Zemicke Van Cittert traduit le degré de cohérence de l'onde laser réfléchie par un tel objet et permet de calculer le rendement d'hétérodynage d'un radar laser cohérent (parfait) sur objet diffusant étendu. Dans le cas, très courant, de radars laser monostatiques, à système d'émission/réception commun, lapplication du théorème de Zemicke Van Cittert montre que ce rendement est de l'ordre de :

$$
\eta_{\text {speckle }} \approx 0,5
$$

\section{Iurbulence atmesphérique}

L'influence de la turbulence provient des déformations en phase provoquées sur l'onde de signal par les inhomogénéités de lindice de l'air. Le paramètre de base le plus utilisé pour caractériser la turbulence et pour en évaluer les effets sur les radars laser cohérents est dénommé "Constante de structure d'indice" $\left(C_{n}\right)$. Il est représentatif des fluctuations de lindice entre 2 points de mesure et permet de définir, pour un site et des conditions météorologiques donnés, le diamètre de cohérence de la turbulence, ro, distance moyenne entre points de l'onde laser dont le degré de cohérence mutuelle est égal à 1/e.

On démontre que la dégradation de signal due à la présence de turbulence peut s'exprimer par :

$$
\eta \text { Turbulence } \approx 1 /\left[1+\left(\Phi_{o p} / r_{0}\right)^{2}\right]
$$

Si le diamètre de l'optique, $\Phi_{\propto p}$ est inférieur au diamètre de cohérence de l'atmosphère, la dégradation des performances du système par la turbulence de l'air est faible. Par contre, il est inutile d'utiliser un diamètre de pupille supérieur à celui de la turbulence (sauf si l'optique du système est adaptative).

La figure 1.4 montre l'évolution du signal hétérodyne en fonction de la dimension de l'optique de réception, pour un diamètre de cohérence donné.

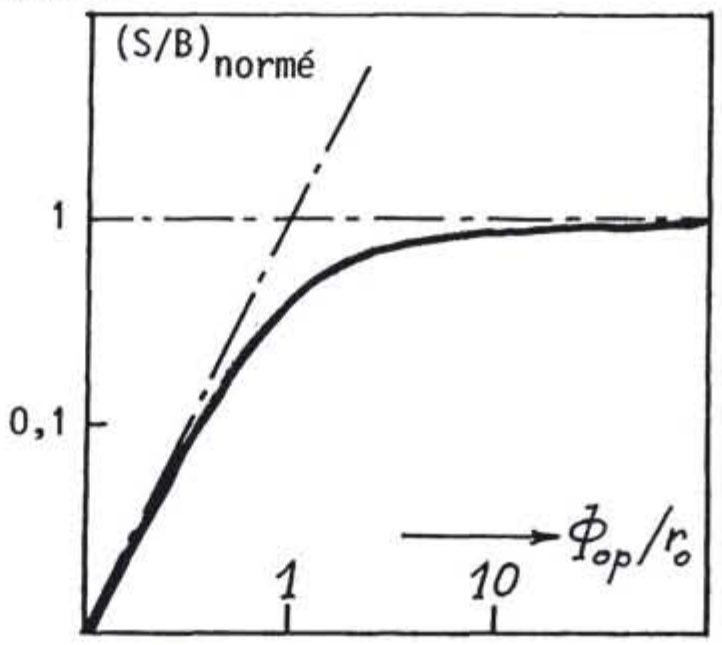

Figure 1-4: Saturation du signal hétérodyne par la turbulence atmosphérique Bendement global d'hétérodynage

Dans la plupart des applications sur objets diffusants étendus, le rendement global d'hétorodynage d'un radar laser cohérent au sol, est de l'ordre de $10 \%$, si l'on prend en compte les dégradations mentionnées ci-dessus. 


\section{CHAPITRE II}

\section{TECHNIQUES DE MESURES}

Les applications essentielles des radars laser se trouvant dans des domaines tels que la défense (télémétrie, guidage), l'espace, la physique de l'atmosphère (sondage de l'atmosphère, mesure de vitesse du vent), l'aéronautique (détection de câbles), il est surtout demandé à ces systèmes des mesures de distances, vitesses ou angles.

Le choix d'une technique est dicté en priorité par la nature du ou des paramètres recherchés, les spécifications en portée et en précision de mesure, l'état de l'art dans les composants de base, etc..., et concerne principalement :

- Le mode d'émission du laser (continu, impulsionnel)

- La modulation du faisceau (en amplitude, fréquence)

- Le mode de détection du signal laser (direct, hétérodyne)

On décrit ci-dessous quelques unes des principales méthodes de mesures mises en oeuvre dans les radars laser, ainsi que les précisions correspondantes.

\section{II.1. TELEMETRIE LASER (Détection directe)}

La télémétrie laser à détection directe se base, dans la plupart des cas, sur la mesure du "temps de vol" d'une impulsion laser pour faire l'aller/retour sur l'objet désigné. Le schéma de la figure Il.1 illustre ce principe, avec les signaux émis et reçus par le dispositif.

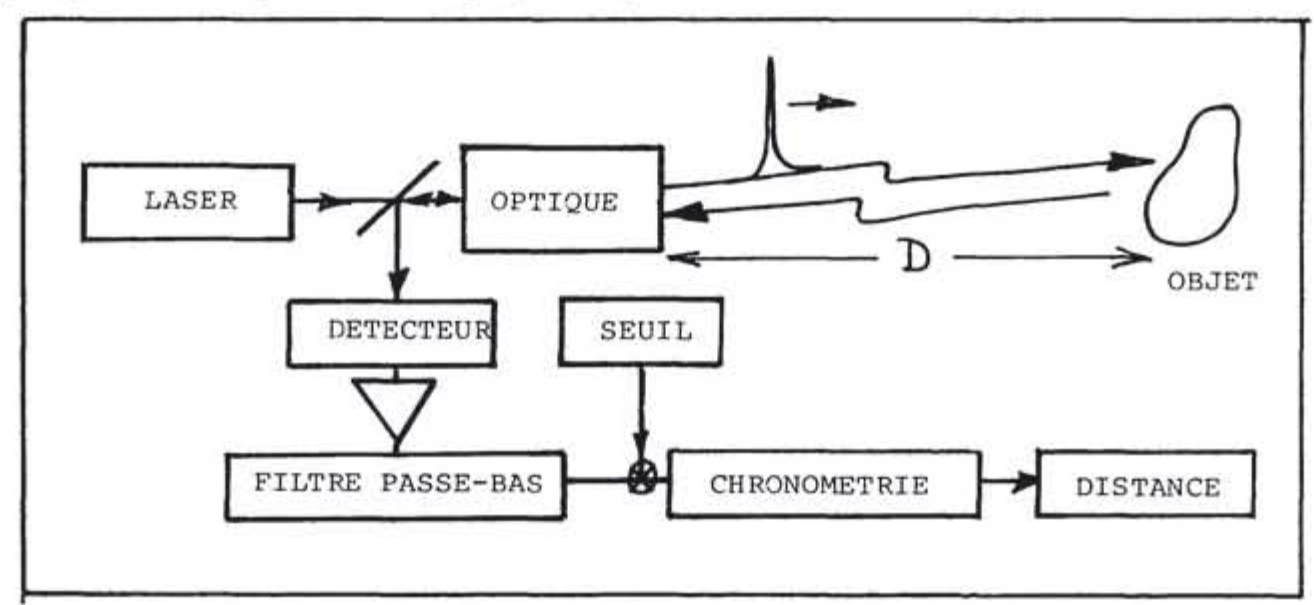

Figure I-1 : Télémétrie impulsionnelle à détection directe

Ce temps de vol taR correspondant à l'aller/retour permet de déduire la distance $d$ de I 'objet par la relation (propagation dans le vide ou dans l'air) :

$$
t_{A R}=2 d / c
$$


Un télémètre laser à détection directe comporte donc généralement une source laser émettant des impulsions brèves (typiquement de 10 à $100 \mathrm{~ns}$ ) à une cadence de quelques tirs par seconde, ou en coup par coup. Une faible fraction du flux émis par le laser est prélevée en sortie de laser et captée par un récepteur dont le signal permet de définir l'instant de départ de l'impulsion.

Le système optique d'émission a pour but de mettre en forme le faisceau pour adapter sa divergence angulaire à la dimension de l'objet. Dans de nombreux cas, cette divergence est limitée à $1 \mathrm{mrd}$, ou moins. Le système optique en réception est chargé de collecter une fraction de flux laser rétroréfléchi par l'objet et de la focaliser sur le photodétecteur. La surface sensible de ce demier est définie de telle sorte que les champs en réception et en émission soient adaptés l'un à l'autre, afin de réduire au plus bas le bruit propre au détecteur. De plus, un filtre interférentiel limite la bande spectrale de fonctionnement du récepteur autour de la longueur d'onde du laser pour optimiser la sensibilité du système.

La chaîne du traitement de signal comporte typiquement les modules suivants :

- Un amplificateur, dont le gain peut être variable dans le temps pour minimiser linfluence de la rétrodiffusion (en particulier la saturation de la chaîne par des échos proches (système optique et particules de l'atmosphère)

- Un filtre adapté à l'impulsion à détecter (dont la bande passante B est inversement proportionnelle à la durée $\tau$ de l'impulsion $(B \sim 0,5 / \tau)$ )

- Un extracteur à seuil et un dispositif de chronométrie, mise en action par l'impulsion laser au départ et arrêtée lors du passage au seuil de l'impulsion détectée.

Les sources laser les plus utilisées actuellement en télémétrie impulsionnelle à détection directe sont les lasers à solide, avec déclenchement électrooptique par cellule de Pockels (Q-Switch). La plus représentative de ces sources est le laser Nd:YAG, pompé optiquement par lampe flash : les impulsions émises $(a) \lambda=1,06 \mu \mathrm{m})$ ont des durées de l'ordre de $\tau \sim 20 \mathrm{~ns}$ et des puissances crêtes de quelques MW.

Les efforts actuels pour l'optimisation des lasers solides concernent, d'une part, l'amélioration de leur rendement grâce au pompage optique par diodes laser (par exemple une partie du spectre d'absorption du Néodyme correspondant au spectre d'émission de diodes telles que Ga Al As : $\lambda \sim 0,808 \mu \mathrm{m}$ ) et d'autre part l'obtention de lasers à sécurité oculaire ("eye safe"), c'est-à-dire de longueur d'onde supérieure à 1,5 $\mu \mathrm{m}$ (limite infrarouge de la courbe de transmission spectrale de l'oeil).

L'absorption de ces rayonnements au niveau de la comée empêche leur focalisation sur la rétine et l'endommagement de cette dernière. Parmi les lasers prometteurs dans ce domaine, on citera les lasers à Erbium et YAG-Raman (laser Nd:YAG translaté en longueur d'onde par effet Raman dans un gaz tel que le méthane) qui émettent à $\lambda=1,54 \mu \mathrm{m}$, ou le laser à Holmium $(\lambda=2,08 \mu \mathrm{m})$.

Parmi les autres sources laser utilisées en télémétrie laser impulsionnelle à détection directe, les lasers $\mathrm{CO}_{2}$, qui émettent un grand nombre de raies entre 9 et $11 \mu \mathrm{m}$, présentent un intérêt particulier par leur adaptation spectrale aux systèmes infrarouges passifs de la bande $8 / 12 \mu \mathrm{m}$ et par leur sécurité oculaire. On citera en particulier les lasers CO2-TEA (à excitation électrique transverse, sous pression atmosphérique) dont les impulsions ont une durée utile de l'ordre de 50 à $150 \mathrm{~ns}$, pour des puissances crête de quelques MW.

Enfin, les lasers semiconducteurs (diodes laser) présentent un intérêt grandissant en télémétrie surtout pour les applications à courtes ou moyennes distances (inférieures à quelques centaines de mètres). 


\section{II.2. MESURE DE VITESSE RADIALE (Détection hétérodyne)}

Le principe général de la mesure de la vitesse radiale d'un objet (composante de sa vitesse le long de l'axe de visée) repose sur le schéma de la figure II.2. Le montage interférométrique correspond à une détection hétérodyne du signal laser ; la source est un laser monomode, continu, stable en fréquence.

La voie oscillateur local, prélevée en sortie de laser par une lame séparatrice, subit une translation en fréquence de valeur $\Delta f$ par rapport au flux laser émis en direction de l'objet.

Après réflexion sur l'objet, le flux laser de retour est lui-même translaté en fréquence, en fonction de la vitesse radiale $V_{R}$ de l'objet (Effet Doppler), d'une quantité :

$$
\Delta f D=2 V_{R} / \lambda
$$

II en résulte que la fréquence du signal hétérodyne délivré par le détecteur après mélange entre flux de signal et oscillateur local est égal à :

$$
f H E T=\Delta f-\Delta f_{D}
$$

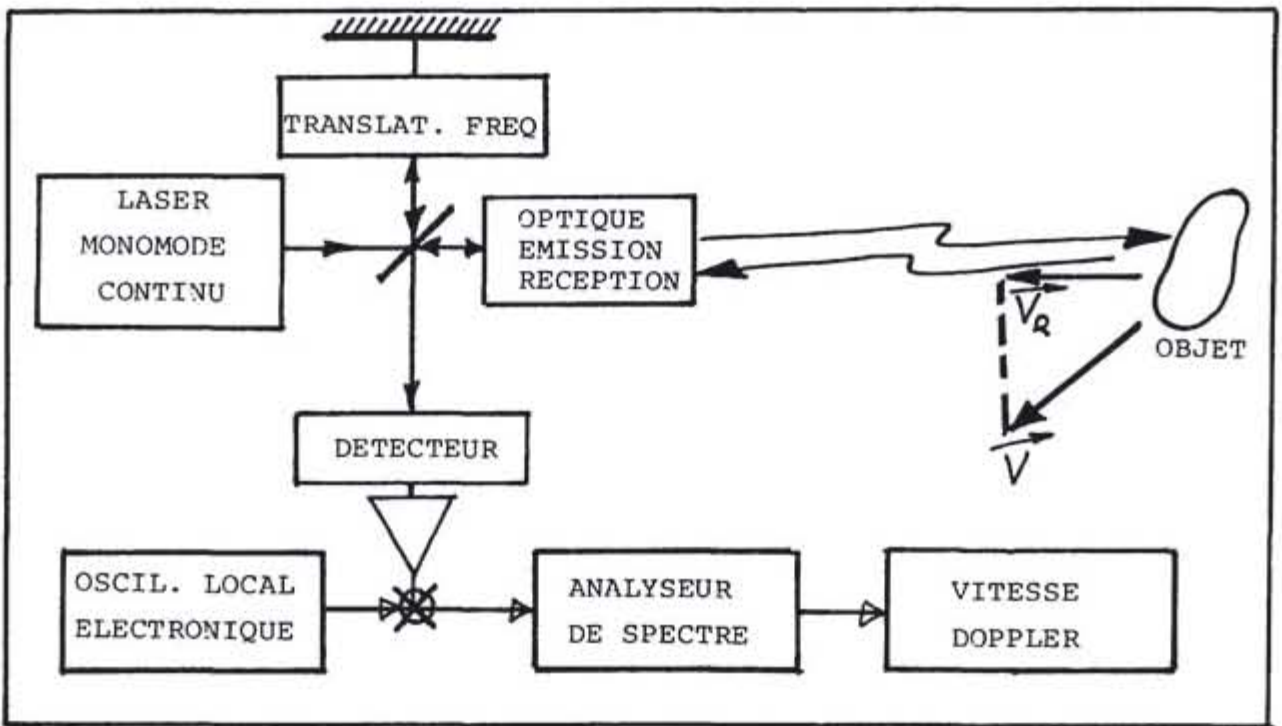

Figure II-2 : Mesure de vitesse Doppler par lidar

La mesure de cette fréquence au moyen d'un analyseur de spectre permet ainsi de déduire la vitesse radiale de l'objet.

II faut noter que la translation de fréquence due à l'effet Doppler est très importante en optique :

- $189 \mathrm{kHz}$ par m/s à $\lambda=10,6 \mu \mathrm{m}$ (laser $\mathrm{CO}_{2}$ ).

- ou $1,89 \mathrm{MHz}$ par $\mathrm{m} / \mathrm{s}$ à $\lambda=1,06 \mu \mathrm{m}$ (laser $\mathrm{Nd}-\mathrm{YAG}$ ),

ce qui confère aux "lidars Doppler" une excellente sensibilité en mesure de vitesse radiale.

Cette sensibilité ou finesse Doppler des radars laser cohérents suppose cependant une très bonne stabilité mécanique de la part du support et des composants du système. Toute vibration, tout mouvement relatif de ce demier par rapport à l'objet (ou de certains composants entre eux) peuvent entraîner des fluctuations dans la mesure. 


\section{II.3. TELEMETRIE DOPPLER (Détection hétérodyne)}

Le paragraphe précédent a rappelé que la mesure de la fréquence du signal hétérodyne donne accès à la connaissance de la vitesse radiale de l'objet. Si cette mesure est effectuée en continu, l'information en distance de l'objet est perdue. Par contre, la détection hétérodyne peut conduire à des mesures simultanées en distance et en vitesse radiale, grâce à 2 types de techniques :

- Emission d'impulsions laser fines

- Emission laser continue et modulation de fréquence

\section{II.3.1. Télémétrie Doppler à impulsions fines}

Le schéma de principe d'un radar laser cohérent à impulsions fines pour télémétrie Doppler se trouve sur la figure II.3

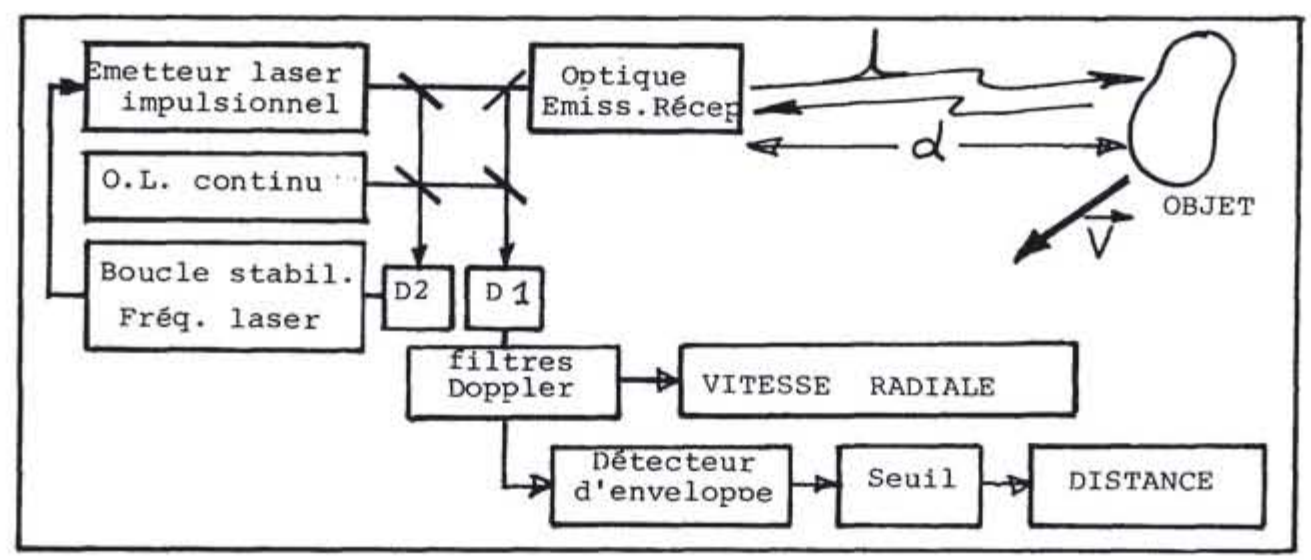

Figure II-3 : Radar laser cohérent à impulsions fines

Les particularités de ce schéma vis-à-vis de celui d'un télémètre à détection directe sont les suivantes :

- L'émetteur impulsionnel est, dans tous les cas, monomode et monofréquence

- Un second laser auxiliaire, continu, monomode et stabilisé en fréquence, tient lieu d'oscillateur local

- Le détecteur à large bande passante, doit être capable de s'adapter aux variations éventuelles de fréquence du signal de battement, dues à l'effet Doppler.

- Le traitement de signal comporte une banque de filtres Doppler et un détecteur d'enveloppe.

Le radar laser cohérent permet ainsi de mesurer à la fois la distance de l'objet par la mesure du temps de vol de l'impulsion et sa vitesse radiale par analyse spectrale du signal hétérodyne.

Les radars laser cohérents à impulsions fines sont actuellement basés sur l'utilisation de lasers $\mathrm{CO}_{2}$-TEA comme émetteurs (avec des lasers $\mathrm{CO}_{2}$ guides d'onde continus comme oscillateurs locaux).

Les lasers $\mathrm{CO}_{2}$-TEA émettent en fait (voir Figure II.4), des impulsions présentant un pic initial fin (50 à $150 \mathrm{~ns}$ ) suivi d'un plateau beaucoup plus long (1 à $2 \mu \mathrm{s})$. Un choix du mélange gazeux $\left(\mathrm{CO}_{2}, \mathrm{~N}_{2}, \mathrm{H}_{2}, \ldots\right)$ dans la cavité permet d'équilibrer les énergies laser contenues respectivement dans le pic et le plateau. Les résultats (voir paragraphe II.5) sur la précision des mesures muntrent que l'optimisation de ces systèmes (en précision) consiste à mesurer la distance de l'objet par détection du pic et la vitesse Doppler par analyse spectrale sur le plateau qui suit. 


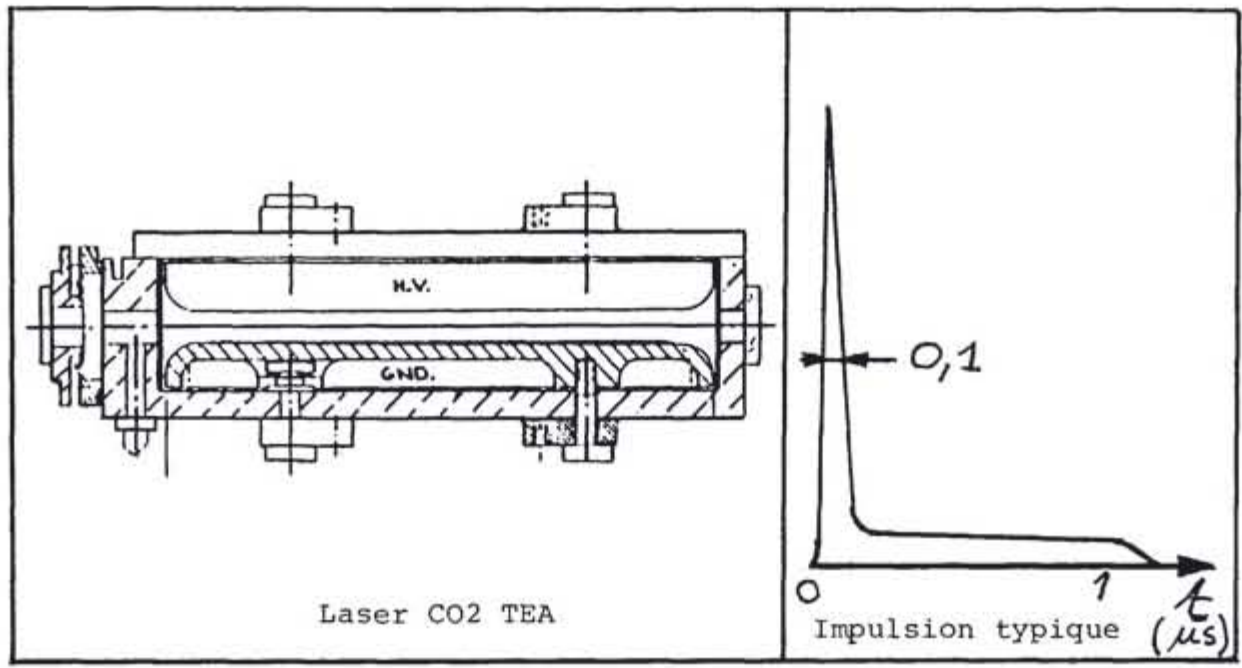

Figure II-4 : Forme typique d'une impulsion laser CO2 TEA

\subsubsection{Télémétrie Doppler à émission laser continue et modulation de fréquence}

D'après la théorie du radar, la distance et la vitesse radiale d'un objet peuvent s'obtenir à partir de l'émission/réception d'une onde électromagnétique quasi continue dans la mesure où celle-ci est codée ou modulée en fréquence à l'émission. Les précisions de mesure sont alors d'autant meilleures que l'émission dure longtemps et couvre un spectre large en fréquence (voir plus loin paragraphe II.5: "Précision des mesures"). Parmi les différentes techniques mises en oeuvre pour optimiser à la fois la précision sur les mesures de distance et de vitesse Doppler, deux sont exposées ci-dessous : la "compression d'impulsion" et la méthode "CTFM".

\section{Compression d'impulsion}

Dans cette méthode (dont le principe est exposé sur la Figure II-5), le faisceau laser est séparé en 2 voies en sortie de l'émetteur (continu, monomode et stable en fréquence). La voie la plus faible (flux de quelques $\mathrm{mW}$ en général) constitue l'oscillateur local et se trouve focalisée sur le détecteur. L'autre voie. représentant la quasi totalité du flux laser émis, est modulée en fréquence sur un domaine de largeur $\Delta f$ : une des lois de modulation les plus utilisées consiste à moduler cette fréquence linéairement en fonction du temps (modulatrice dite "chirp") pendant une durée $T$ et avec une pente $K^{1}$, de telle sorte que la fréquence du faisceau émis par la radar laser est à chaque instant $\mathrm{t}$, égale à :

$$
\begin{aligned}
& f(t)=f(0)+K t \text { (pour } 0<t<T) \\
& \text { avec } K=T / \Delta f
\end{aligned}
$$




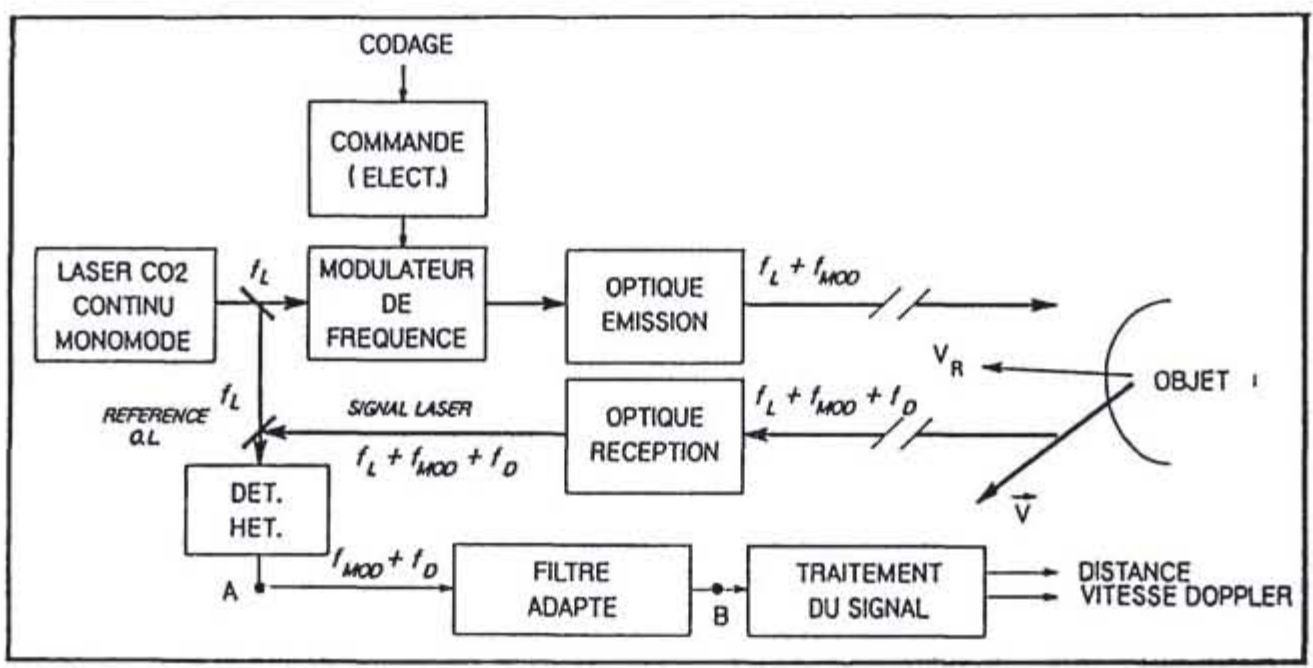

Figure Il-5: Bloo dlagramme d'un lidar à compression d'impulsion

A la réception, le signal électrique hétérodyne créé en sortie de détecteur par le mélange entre l'onde de retour et l'oscillateur local est, lui aussi, modulé linéairement en fréquence pendant la durée $T$ et sa fréquence centrale dépend de la vitesse radiale vR de l'objet visé. Le "filtrage adapté" de ce signal permet de remettre en phiase ses composantes spectrales sous forme d'un pic d'autocorrélation, dont la durée (à $3 \mathrm{~dB}$ ) est approximativement égale à :

$$
\tau \sim 1 / \Delta f
$$

Le signal est ainsi "comprimé" dans le temps d'un rapport Tuf (appelé "taux de compression") vis-à-vis de la durée $T$ de l'émission initiale et il apparait avec un délai t (dû au temps de vol tar aller/retour de la lumière et au temps de transit dans le filtre électronique adapté) égal à :

$$
t=t A R+f_{D} / K
$$

par rapport à la fin de la modulation à l'émission.

Puisque l'instant d'apparition du pic d'autocorrélation du signal dépend à la fois de la distance et de la vitesse radiale de l'objet, une seule mesure ne suffit pas pour déterminer ces 2 paramètres. Pour lever cețte "ambiguité en distance Doppler", le radar laser à compression d'impulsion "chirp" doit émettre sur l'objet 2 ondes successives dans le temps, de même durée T, mais de pentes de modulations différentes. $\mathrm{Si}$, par exemple, ces pentes sont de signes contraires $(+\mathrm{K}$ et $-\mathrm{K})$, les impulsions comprimées correspondantes apparaitront aux instants $\mathrm{t}+$ et $\mathrm{t}$ - tels que :

$$
\begin{aligned}
& t^{+}=t_{A R}+K f_{D} \\
& t^{-}=t_{A R}-K t_{D}
\end{aligned}
$$

La distance $d$ et la vitesse $V_{R}$ radiale de la cible s'obtiennent alors sans ambiguité de ces 2 mesures par la résolution de ce système à 2 équations :

$$
\begin{array}{ll}
\text { tAR }=\left(t^{+}+t\right) / 2 & d=c\left(t^{+}+t\right) / 2 \\
t o=\left(t^{+}-t^{+}\right) / 2 K & \text { VR }=\lambda\left(t^{+}-t\right) / 4 K
\end{array}
$$


Parmi les composants de base d'un lidar à compression d'impulsion (autres que le laser continu monomode et le détecteur hétérodyne), on citera le modulateur de fréquence extracavité, typiquement constitué de cellules de Bragg acoustooptiques (et permettant de moduler la fréquence laser sur quelques $\mathrm{MHz}$ à quelques dizaines de $\mathrm{MHz}$ ), ainsi que le filtre adapté, souvent constitué par des lignes à retard dispersives (par exemple, lignes électroacoustiques de surface), dont les dispersions couvrent quelques dizaines de $\mu \mathrm{s}$. La figure II-6 illustre ces 2 composants.

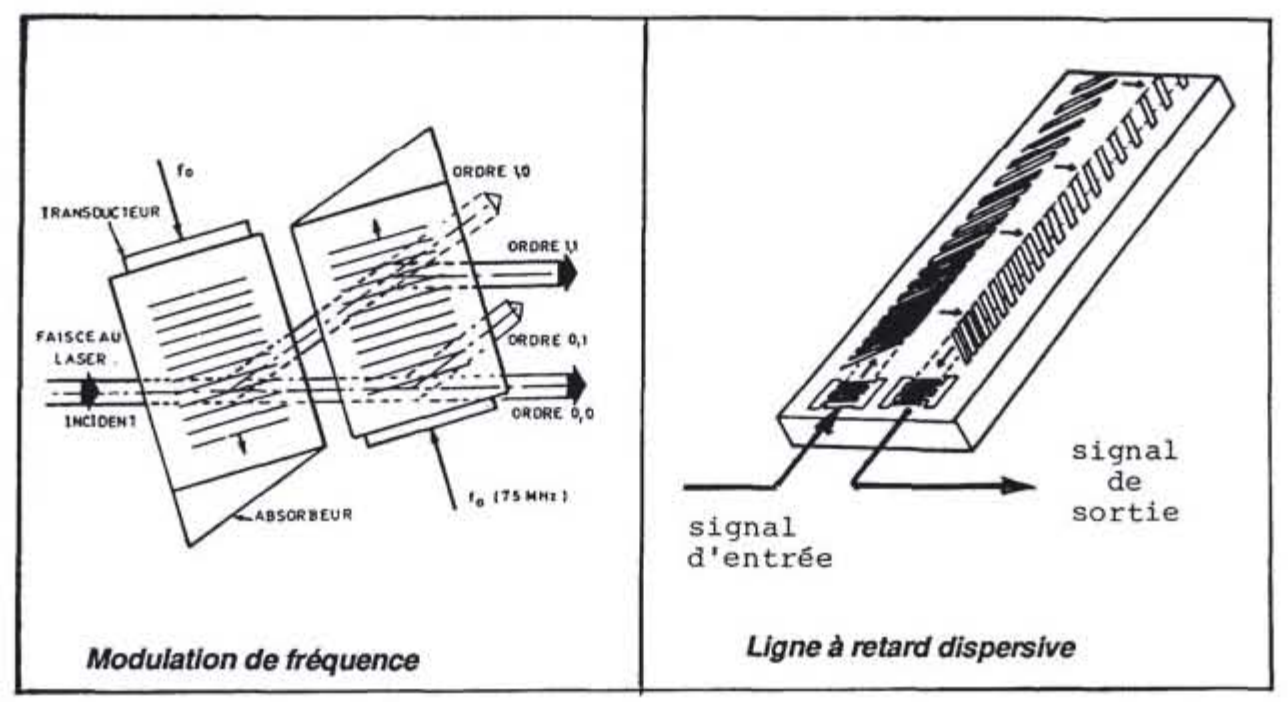

Figure II-6 : Composants d'un lidar à compression d'impulsion

\section{Méthode CTFM}

Le schéma de principe de la métode CTFM (Emission Continue à Modulation de Fréquence ou FMCW : Continuous Wave, Frequency Modulation) est très semblable à celui de la compression d'impulsion (ou C.I.) si ce n'est que la modulation de fréquence, linéaire dans le temps est appliquée directement sur le laser lui-même (modulation intracavité, voir figure II-7) que la fréquence du signal hétérodyne résultant n'est plus modulée en fréquence (comme c'est le cas en C.I.) mais demeure constante tout au long de la mesure. Sa valeur dépend à la fois de la distance et de la vitesse radiale de l'objet et la levée de cette ambiguité "distance/Doppler" s'effectue aussi par 2 mesures successives (par exemple de pentes de modulations opposées", $+K^{\prime}$ et $-K^{\prime}$ ). De même que précédemment, la distance et la vitesse radiale de l'objet sont égales à :

$$
\begin{aligned}
& d=c\left(f^{+}+f\right) / 4 K^{\prime} \\
& v R=\lambda\left(f^{+}-f\right) / 4
\end{aligned}
$$




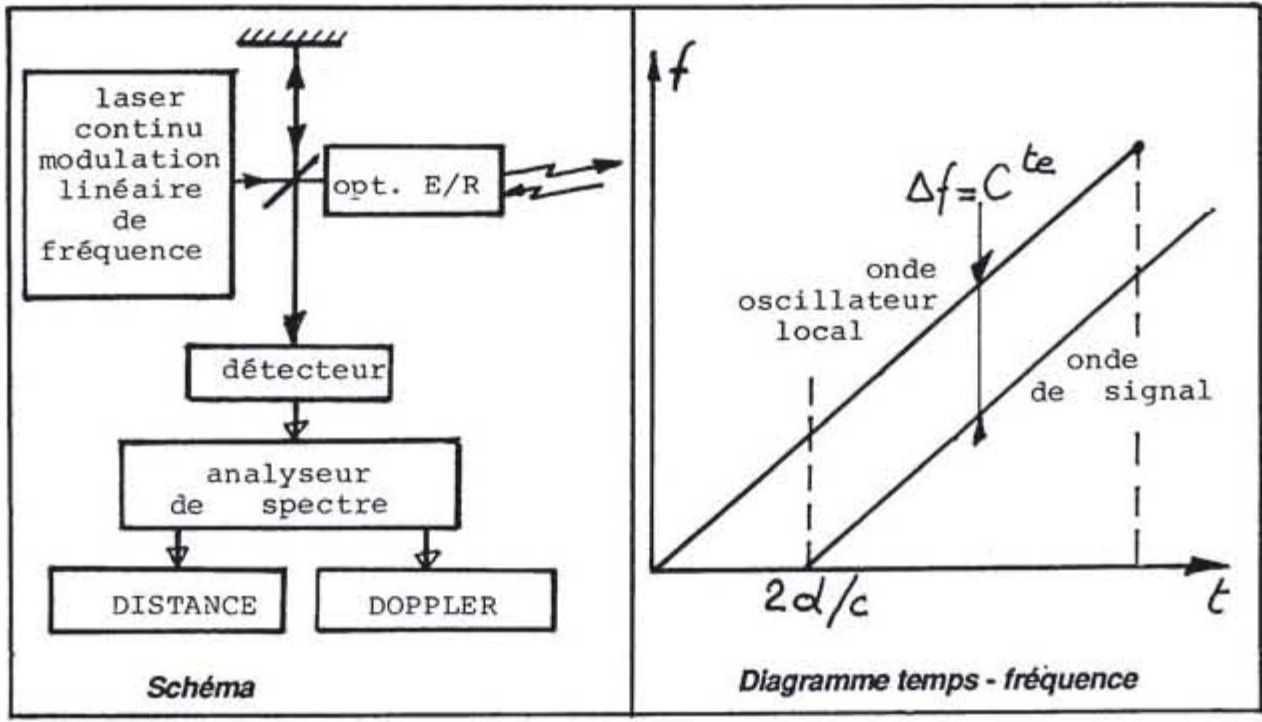

Figure II-7: Principe du mode CTFM

\section{II.4 ECARTOMETRIE LASER (MESURES D'ANGLES)}

Les radars laser peuvent s'utiliser comme écartomètres, c'est-à-dire pour mesurer des angles, en particulier pour le guidage de missiles (autodirecteurs laser), les alignements de systèmes optiques (télécommunications entre satellites), etc...

Le principe de base de ces écartomètres repose sur l'emploi de détecteurs multiples (ou matrices) disposés dans le plan focal (ou sa proximité) d'un système optique de collection de flux laser. La localisation de limpact du faisceau laser sur ces détecteurs (par comparaison de leurs réponses respectives) permet de définir angulairement la direction du faisceau incident par rapport à l'axe optique du système de réception (fig II-8)

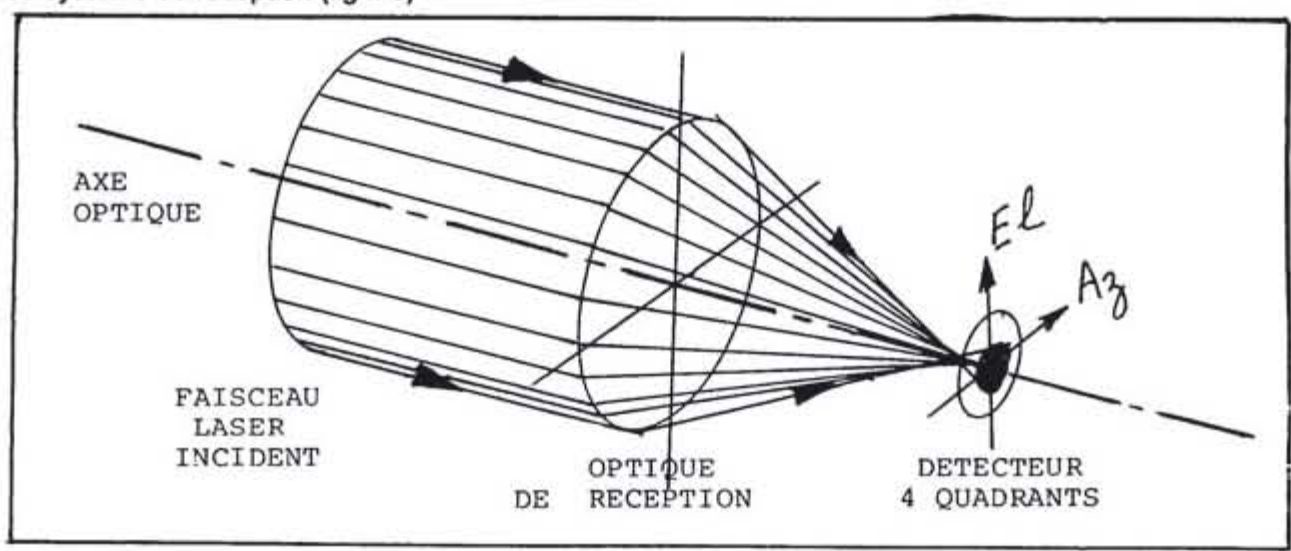

Figure ll-8 Ecartomètre laser 
L'un des dispositifs d'écartométrie laser les plus simples, et communément utilisé, comprend une cellule à 4 quadrants, comportant 4 détecteurs indépendants disposés en croix. Lorsque la direction du faisceau laser est proche de l'axe, la tache laser se répartit sur les 4 détecteurs qui délivrent alors 4 signaux $\mathrm{S}_{1}$. $\mathrm{S}_{2}$, $\mathrm{S}_{3}$ et $\mathrm{S}_{4}$ qui permettent de déduire les coordonnées en azimuth et en élévation de ce faisceau à partir des courbes d'étalonnage angulaire (ou courbes d'écartométrie) $S_{A_{2}}$ et $S_{\Theta l}$ telles que :

$$
\begin{aligned}
& S_{A Z}=\left[\left(S_{2}+S_{4}\right)-\left(S_{1}+S_{3}\right)\right] / S \\
& \text { Sel }=\left[\left(S_{1}+S_{2}\right)-\left(S_{3}+S_{4}\right)\right] / S \\
& \text { avec } S=S_{1}+S_{2}+S_{3}+S_{4}
\end{aligned}
$$

Ces courbes d'écartométrie présentent en général 2 zones : la première, ou zone d'écartométrie linéaire. représente le champ utile de mesure dicté par la dimension relative de la tache laser vis-à-vis de celle de la cellule et par sa loi de répartition en éclairement. La seconde zone, périphérique ou d'acquisition est limitée par la saturation du signal en bord de champ.

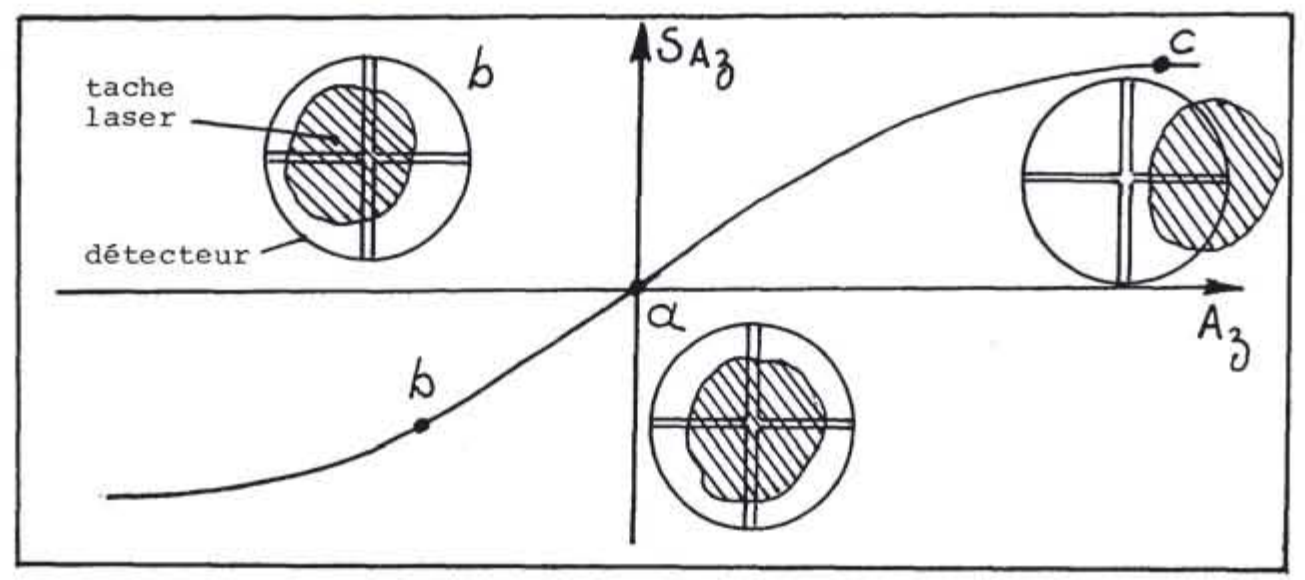

\section{5}

Figure II-9: Courbe d'écartométrie laser

Les précisions de mesure en distances, vitesses radiales ou coordonnées angulaires de cible constituent, avec la portée, les performances de base d'un radar laser. Les limitations des lidars en précision de mesures dans ces trois domaines sont imposées par les résultats issus des theories modernes du radar et du traitement du signal.

Sans entrer dans le détail des calculs, on rappellera seulement que pour une portée donnée, les précisions ultimes sur les mesures de ces 3 paramètres et leurs résolutions sont limitées par la durée $T$ de la mesure, la largeur $\Delta f$ du spectre, la dimension de l'optique $\Phi_{o p}$ et la longueur d'onde d'émission $\lambda$.

- La précision Doppler est d'autant meilleure que la durée du signal est importante et la longueur d'onde du laser faible : $\sigma \mathrm{VR}>\lambda / 2 \mathrm{~T}(2 \mathrm{~S} / \mathrm{B})^{1 / 2}$

- La précision en distance est d'autant meilleure que la largeur du spectre émis est importante, ou que l'impulsion est courte : $\sigma_{d}>c / 2 \Delta f(2 S / B)^{1 / 2}$

- La précision angulaire est d'autant meilleure que l'optique a un diamètre important et que la longueur d'onde est faible : $\sigma_{\theta}>\lambda / \Phi_{0 p}(2 \mathrm{~S} / \mathrm{B})^{1 / 2}$ 


\title{
CHAPITRE III
}

\section{EXEMPLES D'APPLICATIONS DES RADARS OPTIQUES}

\begin{abstract}
Les radars optiques trouvent de plus en plus d'applications dans des domaines aussi divers que le sondage à distance de l'atmosphère (surveillance de l'environnement, détection de polluants, mesure de la vitesse du vent, ...), la sécurité aérienne (aide à la navigation et à l'atterrissage, détection d'obstacles fins et de câbles), ou la conduite de tir (télémétrie, illumination laser de cible, guidage de missiles, acquisition et poursuite de cibles, trajectographie,...).

Ils apparaissent aussi dans des projets de télécommunications spatiales.

Certains ont atteint le stade opérationnel tels que les télémètres ou illuminateurs laser alors que d'autres n'est sont qu'à l'état de maquettes de laboratoire. On donne ci-dessous 3 exemples typiques : un lidar atmosphérique de type DIAL, un lidar pour la détection de câbles et un imageur à laser $\mathrm{CO}_{2}$ pour conduite de tir.
\end{abstract}

\section{III.1. LIDAR ATMOSPHERIQUE}

\section{III.1.1. Généralités}

Les lidars atmosphériques sont destinés au sondage à distance de l'atmosphère pour :

- Mesurer les valeurs de paramètres météorologiques tels que direction et vitesse des vents, humidité, température, pression, ...

- Mesurer les propriétés optiques de composants climatiques (nuages, aérosols) en fonction de l'altitude

- Y détecter la présence éventuelle de composants ; spécifier et mesurer leur concentration (gaz toxiques, polluants, etc...)

Ces applications concernent l'atmosphère dans son ensemble, depuis le sol jusqu'à une altitude de 100 km environ. Les mesures s'effectuent actuellement soit à partir du sol (installations fixes ou mobiles) soit à partir d'avions, mais de nombreux projets de lidars spatiaux, montés sur satellites, sont à l'étude (NASA, ESA, CNES, ...).

Dans la plupart des cas, un lidar atmosphérique émet, dans l'atmosphère, des impulsions lumineuses brèves bien que les particules et molécules de l'air diffusent tout au long du parcours. Une fraction du flux laser réfléchi dans la direction du lidar (ou rétrodiffusé) est recueillie par un télescope, généralement solidaire de l'émetteur. Le signal délivré par le lidar à chaque instant $t$ après l'émission de l'impulsion provient de la tianche d'atmosphère atteinte à linstant t/2 donc située à la distance :

$$
\begin{array}{ll}
\text { et d'épaisseur: } & d=c t / 2 \\
& e=c t / 2
\end{array}
$$

si $\tau$ est la durée de l'impulsion lumineuse érnise. 


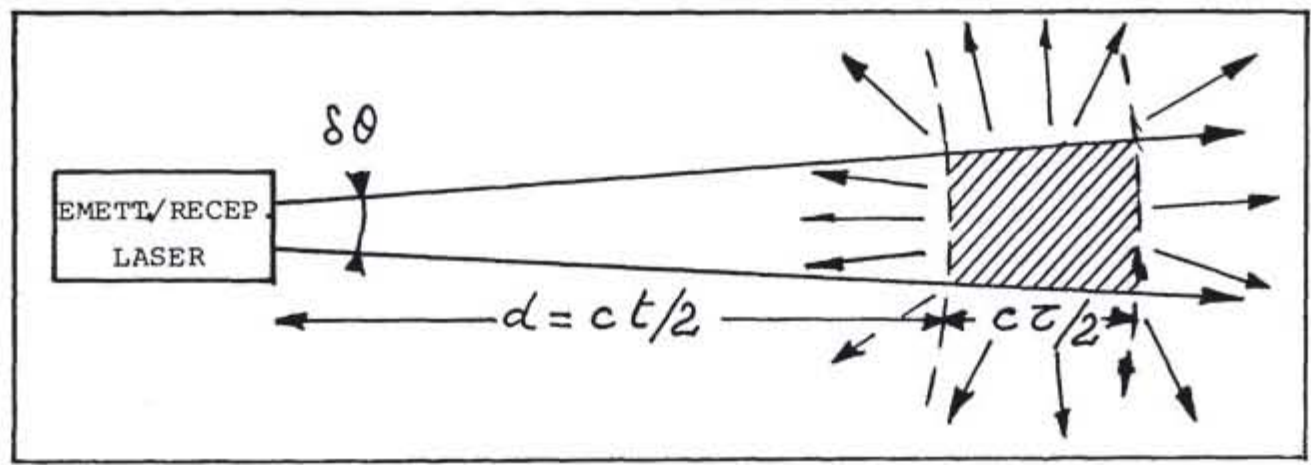

Figure II-1: Volume instantane de sondage par un lidar atmosphérique impulsionnel

Si $\delta \theta$ est la divergence totale du faisceau émis, le signal reçu à chaque instant $t$ après le départ provient d'un volume d'espace de côté (transversal au faisceau) égal à ct $\delta \theta / 2$, de profondeur c $\tau / 2$ et distante de ct/2.

L'intérêt des lidars par rapport aux radars traditionnels ou aux systèmes optroniques passifs est, pour de telles applications, la finesse du volume d'analyse qui, même à grandes distances, peut représenter quelques dizaines de mètres cubes.

Le calcul montre que le flux laser rétroréfléchi dans le système par chaque volume élémentaire d'atmosphère situé à une distance $d$ est de la forme suivante :

$$
F(d)=c E_{i} T_{o p} A_{o p} \beta_{\pi}(d) T_{a t m}(2 d) / 2 d^{2}
$$

où

$$
\begin{array}{ll}
\text { Ei } & \text { :Energie laser émise par impulsion. } \\
\text { Top. Tatm(2d) } & \begin{array}{l}
\text { Transmissions respectives de l'optique et de l'atmosphère sur le parcours } \\
\text { aller/retour }
\end{array} \\
\text { Aop } & \text { :Aire de la pupille de l'optique réceptrice } \\
\beta_{\pi} \text { (d) } & : \text { Coefficient de rétrodiffusion linéique global de l'atmosphère à la distance d. }
\end{array}
$$

\section{III.1.2. Lidar à absorption différentielle (DIAL)}

Un lidar atmosphérique à absorption différentielle, ou DIAL (Differential Absorption Lidar), est destiné à l'analyse des constituants de l'atmosphère et à la mesure de leur concentration. Le principe de la détection d'un constituant par Lidar (Dial) repose sur la brusque et importante variation du coefficient d'absorption spectral de ce constituant à certaines longueurs d'onde (dites de résonnance), caractéristiques de sa composition chimique. Si lon fait ressortir la contribution du constituant analysé dans l'expression de la transmission atmosphérique sur le parcours aller/retour entre le lidar et la tranche d'atmosphère à la distance $d$, on peut écrire que, pour toute longueur d'onde située à proximité de la raie de résonnance (ou sur cette raie) :

$$
T_{\text {atm }}(2 d)=\exp \left[-2 \int_{0}^{d}\left[\beta(z)+\alpha_{d}(z)+\alpha(z)\right] d z\right]
$$

où $\quad \beta(z) \quad$ Coefficient global de diffusion linéique spectral de l'atmosphère

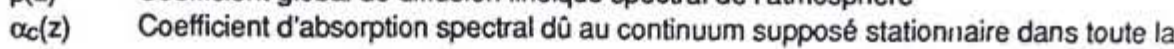




\section{bande spectrale fine centrée sur la résonnance du constituant. \\ $\alpha(z) \quad$ Coefficient d'absorption spectral du constituant recherché}

Si l'une des raies d'émission du laser est exactement centrée sur le pic d'absorption du constituant (longueur d'onde $\lambda \mathrm{N}$ ), il en résulte pour cette raie une chute relative de signal par rapport à la raie située hors résonnance ( $\lambda \mathrm{OFF}$ ), d'autant plus importante que la concentation du constituant est grande. Le schéma de la figure III.2 représente la variation locale de la transmission spectrale de l'atmosphère due à la présence du constituant et l'évolution temporelle des signaux lidars à 2 longueurs d'onde ( $\lambda$ ON et גOFF)

Dans l'hypothèse où la diffusion globale de l'air et l'absorption due au continuum (c'est-à-dire due aux composants autres que le constituant recherché), sont les mêmes à $\lambda$ ON et $\lambda$ OFF, une comparaison de ces 2 courbes permet de déduire à la fois la localisation et la concentration correspondante du constituant (recherche de polluants), par exemple grâce au rapport des flux reçus aux 2 longueurs d'onde :

$$
[F O N(d) / F O F F(d)]=\left[E_{i, O N} / E_{i, O F F}\right] \exp \left[-2 \int_{0}^{d}[\alpha O N(z)-\alpha O F F(z) d z]\right.
$$

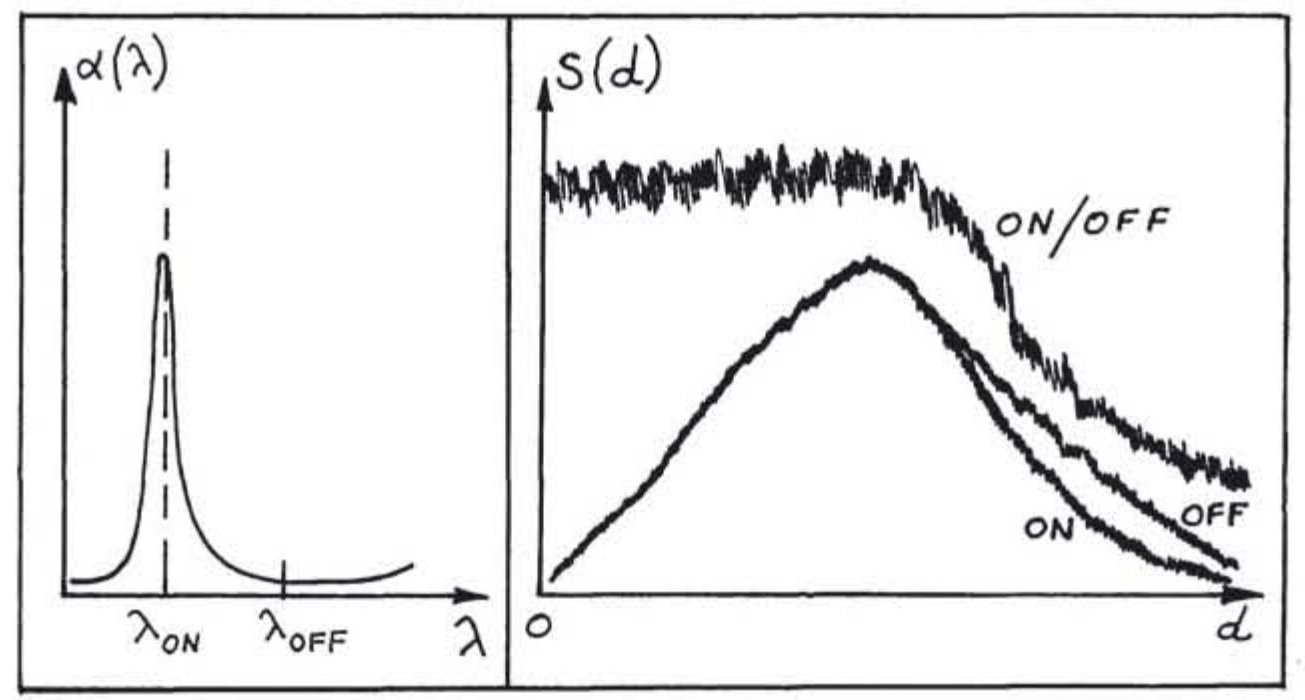

Figure III-2 : Signaux de retour dans un lidar dial

\section{III.1.3. Lidar atmosphérique Doppler}

Un lidar Doppler atmosphérique est destiné à la mesure de la vitesse du vent par détection hétérodyne du par flux laser rétrodiffusé pour les molécules et/ou particules présentes dans l'air et analyse en fréquence du signal (effet Doppler). Ces mesures peuvent s'effectuer à des distances plus ou moins importantes : inférieures à $100 \mathrm{~m}$ pour un hélicoptère, quelques $\mathrm{km}$ pour les mesures de cisaillement de vent sur avion (Windshear, Clean air turbulence).

Le schéma de principe de tels lidars est représenté par la figure III-3, par exemple, dans le cas de mesures à courtes distances. L'optique compor'o un laser monomode continu, stable en fréquence et un 
montage interférométrique : le bras de référence peut être translaté en fréquence grâce à un modulateur acoustooptique (cellule de Bragg), typiquement de l'ordre de $50 \mathrm{MHz}$. Le faisceau d'analyse est focalisé par l'optique d'émission sur une zone đ'atmosphère située à la distance désirée. Grâce à la sélectivité de la détection hétérodyne (théorème de lantenne), seule cette zone participe utilement au signal, les autres plus ou moins défocalisées y contribuant très peu.

Le flux laser rétroréfléchi par les aérosols présents dans le volume de focalisation est translaté en fréquence par l'effet Doppler dô au mouvement radial de ces particules. II est détecté par mélange sur le détecteur avec l'onde de référence et l'analyse spectrale du signal résultant conduit à la mesure de cette vitesse radiale. Des analyseurs de spectre "rapides", par exemple, sur supports électroacoustiques à ondes de surface (Surface Acoustic Wave Devices : SAWD) permettent des précisions de mesures de l'ordre de 10 à $20 \mathrm{~cm} / \mathrm{s}$ (avec un laser $\mathrm{CO}_{2}$ et pour une durée de mesure de quelques $20 \mu \mathrm{s}$ )

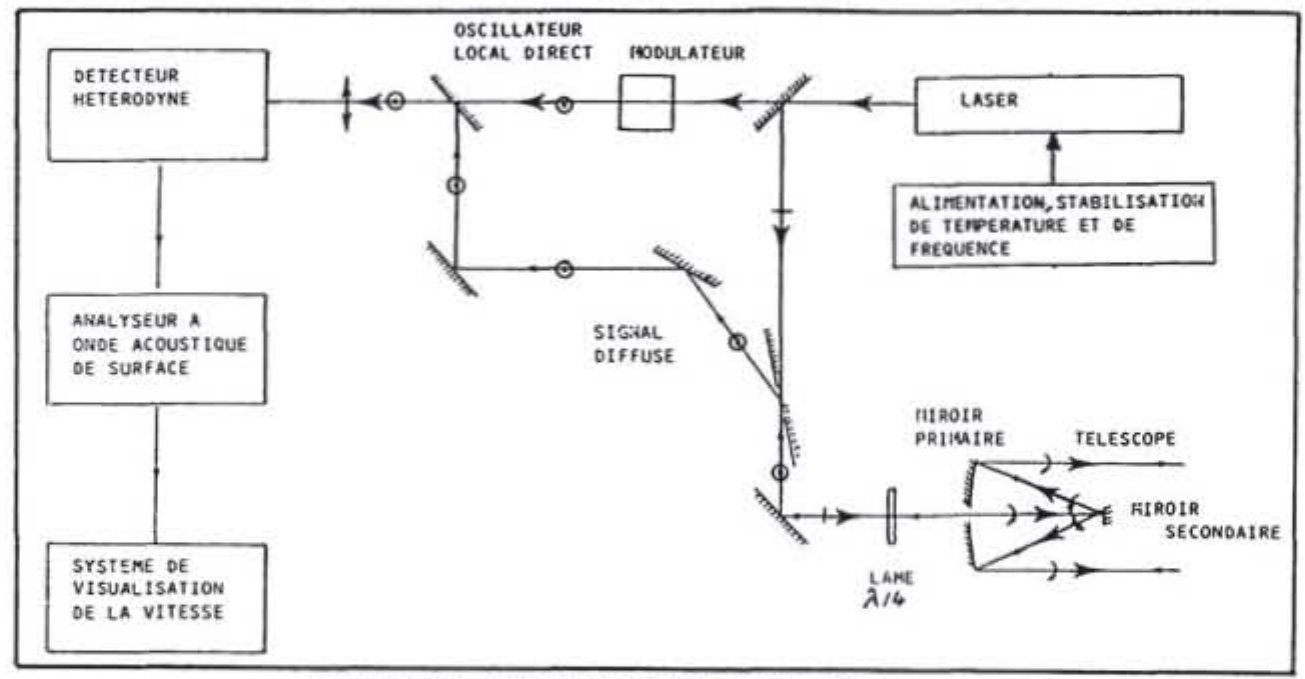

Figure III-3 : Lidar atmosphérique Doppler

Si la distance de mesure est beaucoup plus importante, lutilisation d'un laser impulsionnel comme émetteur s'impose pour des raisons de bilan énergétique, un second laser, continu, servant d'oscillateur local. Dans ces conditions, la précision Doppler est limitée par la durée de limpulsion et les fluctuations de fréquence relatives entre les 2 lasers. Par exemple, avec un laser $\mathrm{CO}_{2}$ (durée d'impulsion $1 \mu \mathrm{s}$ ), la précision Doppler est de quelques $\mathrm{m} / \mathrm{s}$.

Les lasers les plus utilisés par ces applications sont les lasers $\mathrm{CO}_{2}(\lambda=10,6 \mu \mathrm{m})$ et $\mathrm{Ho}: \mathrm{Yag}(\lambda=2,1 \mu \mathrm{m})$

\section{III.2. RADAR LASER POUR DETECTION DE CABLES}

Un second domaine d'utilisations potentielles pour les radars laser est celui de limagerie tridimensionnelle avec, comme applications typiques, la détection d'obstacles fins (cheminées, pylônes, căbles,...) pour avions à très basse altitude, et pour hélicoptères, ou la conduite automatique de robots mobiles.

L'imagerie passive traditionnelle (de type télévision ou infrarouge) présente parfois des difficultés à faire ressortir les obstacles fins ou les détails du relief dont la présence sur la trajectoire constitue la cause de 
trop nombreux accidents, en particulier pour les hélicoptères. Par sa capacité à explorer finement son environnement et grâce a la précision de ses mesures en distance, le radar laser apparait comme un outil de choix dans la détection d'obstacle et, en particulier, celle de cábles. De nombreux programmes de recherche et de développement sont actuellement en cours dans ce domaine.

Le principe de base de tels radars laser s'appuie sur l'association d'un télémètre à haute cadence d'émission et d'un déflecteur de faisceau, chargé de diriger le pinceau laser sur la zone à analyser et d'en assurer le balayage angulaire. Pour chaque direction visée, la mesure de la distance du point d'impact du faisceau laser, associée à la connaissance de ses coordonnées angulaires, permet de constituer une cartographie en 3 dimensions du paysage environnant, de laquelle ressortiront les obstacles éventuels, grâce à leur contraste en distance vis-à-vis du fond (fig III-4).

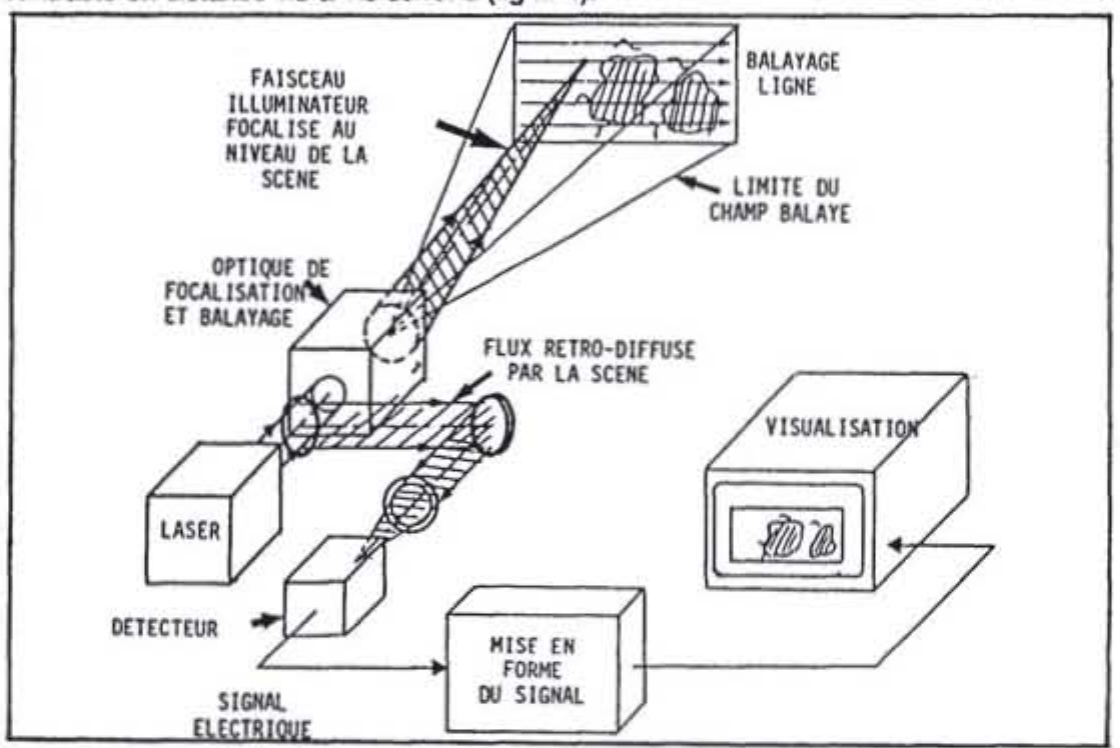

Figure III-4 : Principe de radar iaser pour imagerie tridimensionnelle

Dans le cas particulier de la détection de câbles, la portée de tels systèmes repose essentiellement sur les caractéristiques photométriques des sources laser disponibles et la rétroréflectance apparente des câbles, en fonction de lincidence du faisceau. L'influence de latmosphère est plus ou moins importante, en fonction de la distance de détection exigée ( $<500 \mathrm{~m}$ pour un hélicoptère).

La rétroréflectance apparente d'objets tels que des câbles dépend évidemment de leur géométrie en surface (torsade ou gaine extérieure), de leur diamètre, état de vieillissement. Elle est d'autant plus spéculaire et donc sélective angulairement que la long̣ueur d'onde du radar laser est grande (voir schéma de la figure III-5) pour la comparaison entre diverses longueurs d'onde de lidars et de radars millimétriques), œ qui s'explique par le fait que la rugosité apparente de la surface et, par conséquent, la diffusion du flux laser par cette surface sont d'autant plus importantes que la longueur d'onde incidente est faible. 


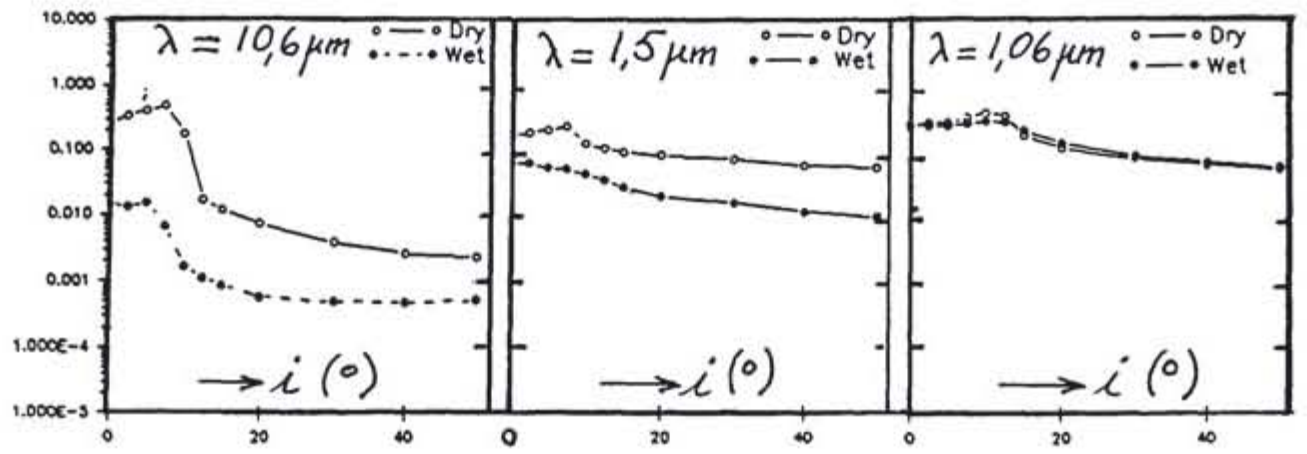

Figure III-5: Reffectance de cábles à diverses longueurs d'onde (en s $r^{-}$

L'une des difficultés de l'imagerie laser "grand champ" provient des limitations en cadence d'émission imposées au laser par le bilan énergétique du système. Cette faiblesse en cadence entraîne, suivant les cas, soit une réduction du champ ou de la cadence image, soit une couverture partielle du champ avec un taux de remplissage et une loi de balayage angulaire adaptés au mieux à l'objet recherché. Par exemple, pour la détection de câbles, on s'appuiera sur la géométrie de ces demiers (câbles = objets filiformes) pour trouver la figure de balayage qui conserve une probabilité importante d'intersection avec un câble tout en minimisant le nombre de points balayés par image.

La figure III-6 illustre ce problème par quelques proportions de balayages curvilignes ne couvrant qu'un faible pourcentage du chamf d'investigation mais avec une forte probabilité d'intersection de l'objet (câble).

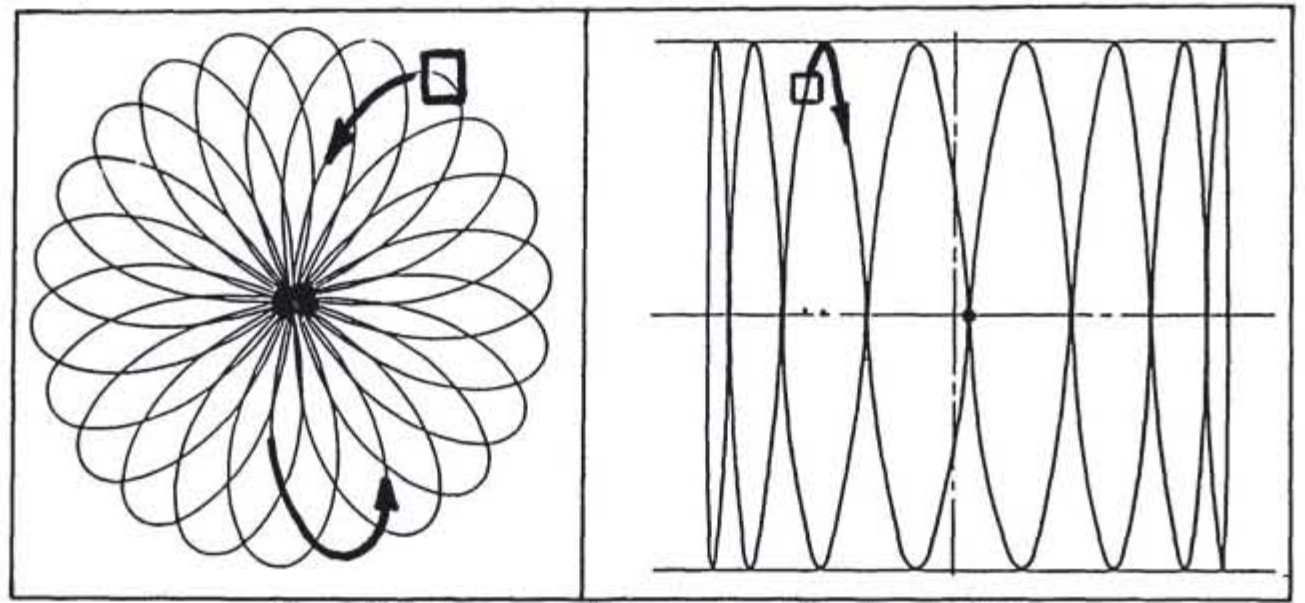

Figure III-6 : Figures de balayage pour détection de cábles

De nombreuses figures de balayage peuvent s'envisager au moyen de prismes toumants (diasporamètres ou prismes de Risley).

En ce qui concerne les lasers envisageables pour de telles applications, on pourra mentionner les diodes laser $(\lambda=0,8-0,9 \mu \mathrm{m})$, les lasers solides pompés par diodes laser à haute cadence (Nd-Yag; Erbium) ou le laser $\mathrm{CO}_{2}(\lambda=10,6 \mu \mathrm{m})$ avec détection hétérodyne. 


\section{III.3. RADAR LASER MULTIFONCTION}

De même que les radars traditionnels ont considérablement évolué, depuis les premiers systèmes destinés essentiellement à des mesures de distances, de même on voit apparaitre des architectures de radars laser destinés à remplir plusieurs fonctions. Cette évolution est rendue possible par les progrès en cours sur les sources laser, elles-mêmes, les diversités de formes d'onde (continue, impulsionnelle, haute ou basse cadence) ou de longueurs d'onde émises.

De nombreux travaux dans $c$ sens s'appuient actuellement sur le laser $\mathrm{CO}_{2} c$ mme émetteur, à cause de la multiplicité de ses formes d'émission, de sa stabilité en fréquence et donc de sa compatibilité avec la détection hétérodyne, origine de nombreux traitements de signal.

Le radar optique est en principe capable de remplir un grand nombre de fonctions attribuées traditionnellement au radar, mais avec des limitations et avantages propres à l'optique. Parmi ces fonctions, on peut citer les suivants :

- Acquisition de cible : imagerie télémétrique, détection de cible mobile

- Identification : imagerie haute résolution, analyse Doppler fine.

- Poursuite automatique

- Aide à la navigation : suivi de terrain, évitement d'obstacles et de câbles

- Anémométrie

- Télécommunications optiques

- Guidage de missiles

Parmi les avantages de l'optique, on citera la résolution angulaire et la sensibilité Doppler, toutes deux dues à la faible longueur d'onde utilisée et qui permettent aux lidars d'accéder à des fonctions d'imagerie fine (en termes de résolution angulaire, en distance ou en vitesse radiale) difficilement accessibles aux radars plus conventionnels.

Les paragraphes ci-dessous montrent quelques résultats obtenus avec un imageur actif basé sur l'utilisation d'un laser $\mathrm{CO}_{2}$ continu monomode de techniques de modulation-démodulation de fréquence et sur la détection hétérodyne du signal laser (résultats Th-CSF).

Le principe d'un tel imageur est illustré sur la figure III-7. Le capteur optique comprend un laser CO2 continu, dont le flux de sortie est séparé en 2 voies : la voie oscillateur local est directement focalisée sur le détecteur hétérodyne et la voie d'émission, modulée en fréquence, puis défléchie angulairement (balayage ligne de type télévision), avant d'être émise sur la scène par l'optique commune en émission et réception. Après réflexion sur la scène, le faisceau laser de retour est focalisé sur le détecteur où il interfère avec l'oscillateur local. Le signal hétérodyne issu de ce mélange est traité dans un filtre adapté et le résultat placé dans une mémoire d'image en fonction des coordonnées angulaires de chaque point visé.

Comme on l'a vu plus haut (paragraphe II.3), la modulation en fréquence du laser peut s'appliquer soit sur la voie d'émission seule (mode dit de "compression d'impulsion") du faisceau, soit intracavité (mode "CTFM"). 


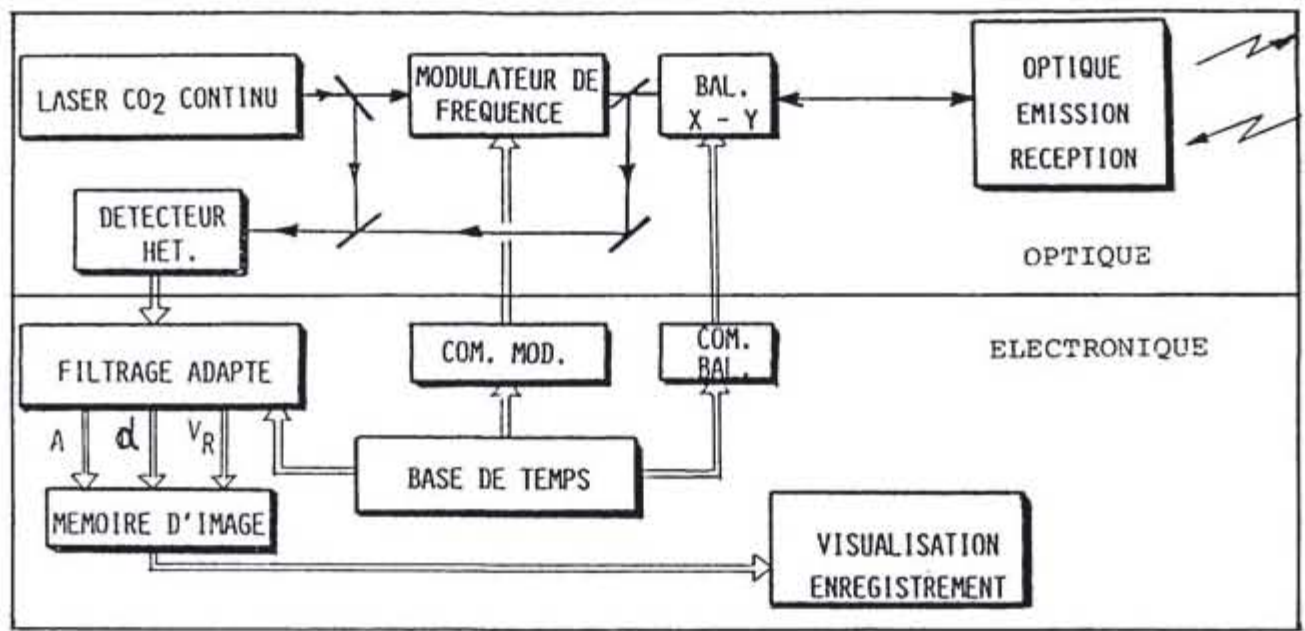

Figure II-7: Principe d'un lidar imageur à modulation de fréquence

Avec un laser continu, de puissance à l'émission de l'ordre de $5 \mathrm{~W}$, une pupille d'émission/réception de 90 $\mathrm{mm}$ de diamètre, ce radar laser effectue des imageries de scènes en réflectance distance et Doppler sur un champ de $190,5^{\circ}$ avec une résolution angulaire de $0,15 \mathrm{mrd}(64 \times 128$ points), et une portée de plusieurs kilomètres de taux de compression utilisé de 400 ( $T=20 \mu \mathrm{s}, \Delta \mathrm{f}=20 \mathrm{MHz}$ ) lui confère des précisions de mesure en distance et en vitesse radiale respective de $+-5 \mathrm{~m}$ et $+-0,5 \mathrm{~m} / \mathrm{s}$. Les figures III-8 et III-9 illustrent les performances de ce lidar, par des images laser en réflectance (pylone et câbles), distance (véhicules) et Doppler (piétons, hélicoptère).

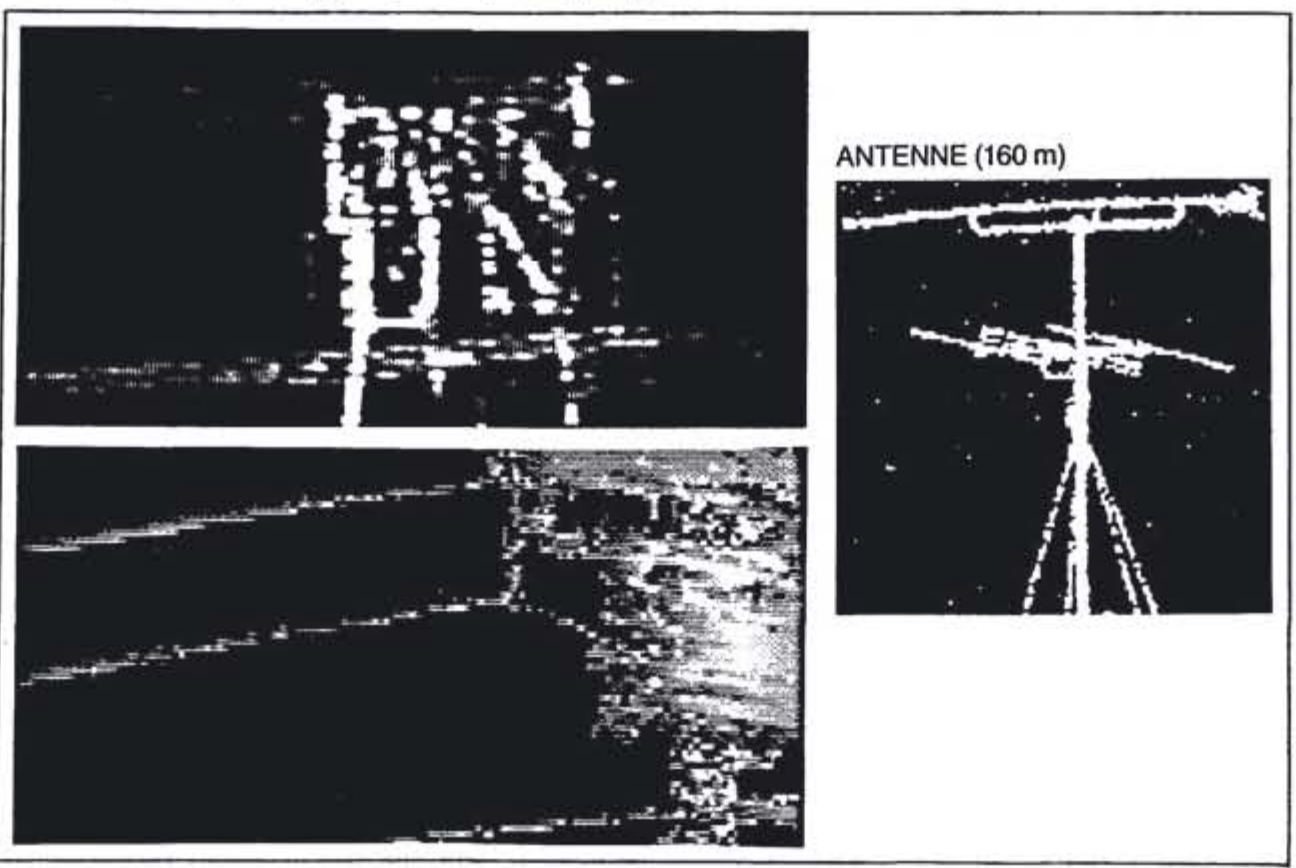

Figure III-8 : Imagerie laser de cábles 


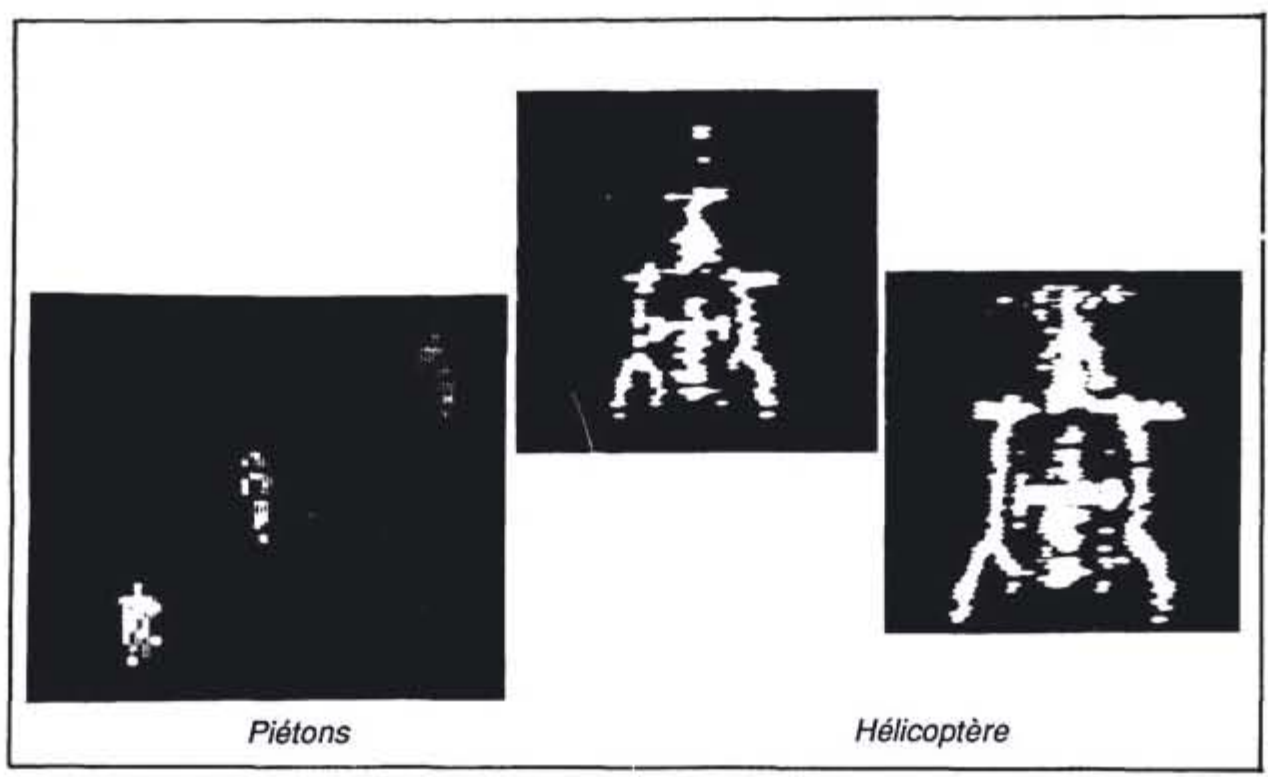

Figure III-9 : Imagerie Doppler

\section{REFERENCES}

1) SPIE Proc. Vol 300 Physics and Technology of Coherence Infra Red Radar. Conference, San Diego, Calif. USA (1981)

2) SPIE Proc. Vol 415, Coherent Infra Red Radar, Systems and applications, Conference, Arlington, Va, USA (1983)

3) Introduction to Sensor Systems

S.A. Hovanessian, Artech House, 1988

4) Télémétrie et transmission d'information à $10.6 \mu \mathrm{m}$ en superhétérodyne G. de Corlieu et J.L. Meyzonnette

Revue Technique - Thomson-CSF Vol 17, n², juin 1985

5)) SPIE Proc. Vol 783, Laser Radar Systems

Conference, Boston, Mass, USA (1987)

6) Physique et théorie du Radar

J. Darricau

Edition SA Deniaud

7) Rarlars, Bases modernes

M. Carpentier

Masson, 1977

8) Radar Hand book

M. Skolnik

Mc Graw Hill, 1970 